\title{
Sodium Deprivation and Salt Intake Activate Separate Neuronal Subpopulations in the Nucleus of the Solitary Tract and the Parabrachial Complex
}

\author{
JOEL C. GEERLING AND ARTHUR D. LOEWY* \\ Department of Anatomy and Neurobiology, Washington University School of Medicine, \\ St. Louis, Missouri 63110
}

\begin{abstract}
Salt intake is an established response to sodium deficiency, but the brain circuits that regulate this behavior remain poorly understood. We studied the activation of neurons in the nucleus of the solitary tract (NTS) and their efferent target nuclei in the pontine parabrachial complex $(\mathrm{PB})$ in rats during sodium deprivation and after salt intake. After 8-day dietary sodium deprivation, immunoreactivity for c-Fos (a neuronal activity marker) increased markedly within the aldosterone-sensitive neurons of the NTS, which express the enzyme 11- $\beta$-hydroxysteroid dehydrogenase type 2 (HSD2). In the PB, c-Fos labeling increased specifically within two sites that relay signals from the HSD2 neurons to the forebrain-the pre-locus coeruleus and the innermost region of the external lateral parabrachial nucleus. Then, 1-2 hours after sodiumdeprived rats ingested salt (a hypertonic 3\% solution of $\mathrm{NaCl}$ ), c-Fos immunoreactivity within the HSD2 neurons was virtually eliminated, despite a large increase in c-Fos activation in the surrounding NTS (including the A2 noradrenergic neurons) and area postrema. Also after salt intake, c-Fos activation increased within pontine nuclei that relay gustatory (caudal medial PB) and viscerosensory (rostral lateral PB) information from the NTS to the forebrain. Thus, sodium deficiency and salt intake stimulate separate subpopulations of neurons in the NTS, which then transmit this information to the forebrain via largely separate relay nuclei in the PB complex. These findings offer new perspectives on the roles of sensory information from the brainstem in the regulation of sodium appetite. J. Comp. Neurol. 504:379-403, $2007 . \quad \odot 2007$ Wiley-Liss, Inc.
\end{abstract}

Indexing terms: sodium appetite; salt appetite; aldosterone; area postrema; ingestive behavior; mineralocorticoid; nucleus tractus solitarius; thirst; lateral parabrachial nucleus; pre-locus; pre-locus coeruleus

Dietary sodium deficiency causes a gradual reduction in blood volume and an impaired ability to increase the overall volume of extracellular fluid. Restoration of blood volume requires the consumption of sodium in addition to water (Stricker and Jalowiec, 1970; Nose et al., 1988). Accordingly, after prolonged hypovolemia animals exhibit a dramatic change in appetite, during which they will seek and ingest unusually large amounts of salt. Sodium appetite, as this behavioral drive is known, has been a subject of continued investigation over the 70 years since it was first demonstrated (Richter, 1936), but the underlying brain circuits remain poorly understood.

Most current ideas about the brain sites responsible for detecting sodium deficiency and stimulating this appetite are derived from studies that involve large brain lesions or intracerebral injection of pharmacological agents (Wolf, 1964, 1967; Contreras and Stetson, 1981; Denton, 1982;

Grant sponsor: National Institute of Heart, Lung, and Blood of the NIH Grant number: HL-25449 (to A.D.L.); Grant sponsor: American Heart Association; Grant number: Predoctoral Fellowship Award 0510050Z (to J.C.G.).

*Correspondence to: Arthur D. Loewy, PhD, Dept. of Anatomy and Neurobiology, Box 8108, Washington University School of Medicine, $660 \mathrm{~S}$. Euclid Ave., St. Louis, MO 63110. E-mail: loewya@pcg.wustl.edu

Received 5 October 2006; Revised 17 May 2007; Accepted 11 June 2007 DOI 10.1002/cne.21452

Published online in Wiley InterScience (www.interscience.wiley.com). 
Stricker and Verbalis, 1996; Johnson and Thunhorst, 1997; Johnson et al., 1999). It is frequently asserted that the stimulation of sodium appetite in response to sodium deficiency is somehow mediated by neurons lying along the anterior wall of the third cerebral ventricle, in or near a region of the hypothalamus that is critically involved in the stimulation of thirst (Johnson and Thunhorst, 1997; Daniels and Fluharty, 2004). Nevertheless, experimental lesions in rats and sheep have consistently failed to establish a necessary role for sites in this region of the brain (Bealer and Johnson, 1979; De Luca et al., 1992; Weisinger et al., 1993; Denton et al., 1996; Fitts et al., 2004). These and other findings have led to the suggestion that sodium appetite is also driven by brain site(s) distant from the ventricular wall of the anterior hypothalamus (Denton et al., 1996).

Because this appetite characteristically increases following a prolonged systemic deficiency (hypovolemia), viscerosensory neurons in the dorsomedial medulla are in an ideal position for transmitting information about sodium balance. However, lesion studies have not established a critical role for this region either. For example, destruction of arterial baroreceptor nerves produces a partial reduction in sodium appetite (Thunhorst et al., 1994), whereas small lesions, placed where these nerves synapse in the dorsolateral nucleus of the solitary tract (NTS), had no effect (Schreihofer et al., 1999).

In fact, a generalized inhibitory role has been ascribed to viscerosensory input signals that ascend from the NTS and from its neighboring circumventricular organ, the area postrema (AP) (Johnson and Thunhorst, 1997). This view is based largely on the massive increase in salt intake that develops after the targeted destruction of the AP in rats (Contreras and Stetson, 1981; Watson, 1985; Edwards et al., 1993; Wang and Edwards, 1997; Curtis et al., 1999). Consistent with this view, sodium appetite can be increased or prolonged by injecting various neurotransmitter antagonists into the lateral parabrachial nucleus (LPBN), a region of the dorsolateral pons targeted by efferent axons from the NTS and AP (Menani et al., 1996, 1998; Menani and Johnson, 1998). Here, relay nuclei in the pontine parabrachial complex (PB) transmit viscerosensory information from the AP and NTS to autonomic and appetite-related sites in the forebrain (Saper and Loewy, 1980; Fulwiler and Saper, 1984; Herbert et al., 1990).

However, potentially contradicting the idea that sodium-related signals from the brainstem are exclusively inhibitory toward salt intake, we identified a small subpopulation of neurons within the NTS that are selectively activated during prolonged sodium deprivation (Geerling et al., 2006a; Geerling and Loewy, 2007). These neurons express the mineralocorticoid receptor as well as the glucocorticoid-inactivating enzyme 11- $\beta$-hydroxysteroid dehydrogenase type 2 (HSD2), which makes them uniquely sensitive to aldosterone (Geerling et al., 2006b). The HSD2 neurons are activated in parallel with sodium appetite under a variety of experimental paradigms (Geerling et al., 2006a; Geerling and Loewy, 2007). In combination with their sensitivity to aldosterone, which selectively enhances salt ingestion (Rice and Richter, 1943; BraunMenendez, 1950; Wolf, 1965; Wolf and Handal, 1966), this finding suggested that they play an important role in driving salt intake.
Based on these unique features, we proposed that the HSD2 neurons receive neural and humoral signals that arise during prolonged sodium deficiency (including elevated levels of aldosterone), and then transmit this information rostrally, to forebrain sites that regulate sodium appetite (Geerling et al., 2006a). Previously, we conducted a series of anterograde and retrograde neural tracing experiments to learn more about the output network of the HSD2 neurons. These experiments showed that the axons of HSD2 neurons in the NTS primarily target two sites in the dorsolateral pons-the pre-locus coeruleus (pre-LC) and the inner division of the external lateral parabrachial subnucleus (PBel-i) - as well as a small site within the basal forebrain, the ventrolateral bed nucleus of the stria terminalis (BSTvl; Geerling and Loewy, 2006b).

In the present set of experiments we tested the possibility that separate signals for sodium need and salt detection are transmitted to the forebrain from the HSD2 neurons and from neighboring neurons in the NTS/AP via separate relay nuclei in the pontine $\mathrm{PB}$ complex. We used the neuronal activity marker c-Fos (Sagar et al., 1988; Dragunow and Faull, 1989; Hoffman et al., 1993) to explore the functional relationship between the HSD2 neurons and their efferent target sites in the dorsolateral pons. We hypothesized that, during sodium appetite, neuronal activation would increase not only in the HSD2 neurons, but also in the specific pontine nuclei that receive their axonal projections.

To produce sodium appetite and HSD2 neuronal activation, we used dietary sodium deprivation-a potent, noninvasive stimulus that selectively stimulates salt ingestion in rats (Wagman, 1963; Contreras and Hatton, 1975; Prakash and Norgren, 1991; Stricker et al., 1991; Lane et al., 1997; Geerling et al., 2006a). Additional groups of sodium-deprived rats were allowed to drink concentrated saline in order to explore the possibility that salt intake stimulates other neurons in the NTS and AP, which transmit feedback signals to the forebrain through separate relay nuclei in the pons.

\section{MATERIALS AND METHODS}

All experimental protocols were approved by the Washington University School of Medicine Institutional Animal Care and Use Committee and conformed to NIH guidelines.

\section{Sodium deprivation and subsequent salt access}

Adult male Sprague-Dawley rats $(\mathrm{n}=19,256-308 \mathrm{~g}$; Harlan, Indianapolis, IN) were group-housed for at least 3 days upon arrival, during which time they were provided ad libitum access to tap water and standard rat chow (PicoLab rodent diet \#20, containing $0.33 \%$ sodium; LabDiet, Richmond, IN). Rats were then separated into individual cages for a baseline period of 6 days prior to sodium deprivation. During this baseline period a low-sodium chow $(0.01 \% \mathrm{Na}$, \#85292, Harlan-Teklad; Madison, WI) was available ad libitum, in addition to two graduated drinking tubes-one containing $\mathrm{dH} 2 \mathrm{O}$ and the other containing concentrated saline $(3 \% \mathrm{NaCl}, 0.5 \mathrm{M})$.

A schematic of the following experimental protocols is provided in Figure 1. To begin the 8-day sodium deprivation period, the saline drinking tube was removed from 


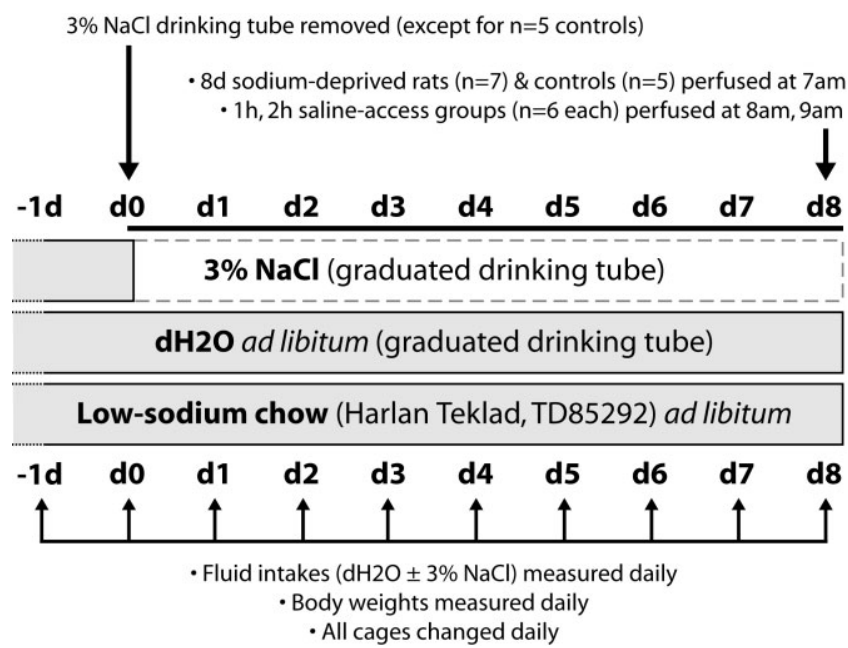

Fig. 1. Experimental protocol for 8-day dietary sodium deprivation, with or without subsequent 1-2 hour access to concentrated saline (note: $3 \% \mathrm{NaCl} \approx 0.5 \mathrm{M}$ ). See Materials and Methods for detailed information.

each cage. Body weights and fluid intake volumes were measured daily (beginning 24 hours prior to removal of saline, so as to include a 1-day baseline value), and clean cages were provided every day to minimize the ingestion of any excreted sodium.

Throughout these experiments an automated 12/12hour light-dark schedule was used (lights-on at 6:30 AM; lights-off at 6:30 PM). At the end of the 8th day after saline removal, the 19 sodium-deprived rats were divided into three groups. Rats in the first group $(\mathrm{n}=7)$ were perfused at 7 am with no access to saline.

At the same time, a graduated drinking tube containing $3 \% \mathrm{NaCl}$ was returned to each rat in the other two groups (with continual access to $\mathrm{dH} 2 \mathrm{O}$ ), which were perfused either 1 hour $(n=6)$ or 2 hours $(n=6)$ later. Their fluid intake volumes (both $\mathrm{dH} 2 \mathrm{O}$ and $3 \% \mathrm{NaCl}$ ) were measured at 30-minute intervals until perfusion. Each rat was left undisturbed until it was anesthetized immediately prior to perfusion. Due to the time required for anesthesia and perfusions, actual saline-access times ranged from 1-1.5 hours in the " $1 \mathrm{~h}$ " group, and $2-2.5$ hours in the " $2 \mathrm{~h}$ " group.

Controls ( $\mathrm{n}=5,267-290 \mathrm{~g}$ adult male Sprague-Dawley rats) were run on a separate week and treated similarly (including daily cage changes and measurements of dH2O and $3 \% \mathrm{NaCl}$ intakes), except that the $3 \% \mathrm{NaCl}$ drinking tube remained continuously available up to the time of perfusion. All rats in these experiments gained weight steadily and similarly during the 8-day experimental period; no significant overall difference in daily body weight gain was evident between the sodium-deprived and control cases $(3.86 \pm 0.23 \mathrm{~g} / \mathrm{d}$ sodium-deprived; $3.28 \pm 0.21$ g/d controls; $P=0.22$ by 2 -tailed $t$-test).

All perfusions and drinking tests were performed early in the morning (shortly after 6:30 AM, the end of the 12-hour dark period in which rats are primarily awake) because, in our experience, both salt intake and c-Fos activation within most brain sites are reduced (and more variable) if measured later in the day, after rats have spent the majority of their time sleeping (see also Chae and Heideman, 1998). The earlier times used for perfusion and testing are probably responsible for the increased percentages of c-Fos-activated HSD2 neurons and the larger average saline intakes (described below) measured in the present study, relative to the values we reported previously after perfusions later in the morning (Geerling et al., 2006a).

\section{Perfusions and histology}

At the end of every experiment rats were anesthetized with pentobarbital $(50 \mathrm{mg} / \mathrm{kg}$ i.p. $)$ and perfused with saline and $4 \%$ paraformaldehyde, as described previously (Geerling and Loewy, 2006b). After overnight fixation in the same solution, brains were divided into separate blocks for sectioning of 1) the medulla (medullary blocks, in every case in these experiments, were promptly cut and immunostained beginning the morning after perfusion), and 2) the pons-forebrain (these brain blocks remained in fixative until they were cut and immunostained within 1-2 weeks after perfusion, performed in batches of $n=2$ per day, as described below). The 50- $\mu$ m-thick frozen sections were cut in the transverse (coronal) plane and loaded immediately into primary antibody solution.

From the medullary block, in every case in this study, one 1-in-5 series of sections through the caudal NTS (from $500 \mu \mathrm{m}$ rostral to the AP extending back to the spinomedullary transition) was labeled for c-Fos using diaminobenzidine (DAB) colorimetric development using protocols described previously (see Geerling and Loewy, 2006b). In all cases the final $\mathrm{DAB}$ reaction time was restricted as closely as possible to 5 minutes in order to minimize variability in intensity and background (actual range 4-6 minutes, due to the amount of time required for transferring large numbers of sections).

Also, in every case an adjacent 1-in-5 series of sections was double-labeled for c-Fos + HSD2 using a doubleimmunofluorescence procedure (see Geerling et al., 2006a). An additional 1-in-5 series of sections was doublelabeled for c-Fos + TH (tyrosine hydroxylase, a marker for catecholaminergic neurons, specifically the A2 noradrenergic group that lies lateral to the HSD2 neurons in the NTS) in every case from the 8-day sodium-deprived, $2 \mathrm{~h}$ saline-access, and control groups.

In order to minimize systematic biases that could arise from histochemical processing, medullary tissue sectioning and immunostaining were initiated simultaneously for all cases in the three experimental groups $(n=19)$ on the day after perfusion using the same histochemical reactions, incubation times, and reagents. Tissue from subsequent control cases $(n=5)$ was processed together in a separate batch using the same immunostaining protocols.

For HSD2 immunofluorescence staining, an affinitypurified sheep polyclonal antibody was used (product 1296; Chemicon International, Temecula, CA). This antiserum was raised against a recombinant protein generated from nucleotides 385-1204 of the rat HSD2 gene (see Gomez-Sanchez et al., 2001). A dilution of 1:40,000 produces optimal signal-to-noise for staining the restricted group of cytoplasmic-immunoreactive neurons located in the NTS (the subject of the present study), as well as cells in the subcommissural organ and neurons in the ventrolateral division of the ventromedial hypothalamic nucleus (Geerling et al., 2006b), in agreement with a prior local- 
ization of HSD2 mRNA by in situ hybridization (Roland et al., 1995).

For the detection of TH we used an affinity-purified sheep polyclonal antibody raised against $\mathrm{TH}$ from rat pheochromocytoma (AB1542, Chemicon; diluted 1:1,000). As noted previously (Geerling et al., 2006b; Geerling and Loewy, 2006b), this antibody only labeled neurons in the well-established distributions of catecholaminergic groups in the dorsomedial and ventrolateral medulla (A1 and A2 noradrenergic; $\mathrm{C} 1, \mathrm{C} 2$, and $\mathrm{C} 2 \mathrm{~d}$ adrenergic groups; and a dense group of TH-immunoreactive (-ir) cells within the area postrema). For $\mathrm{TH}+\mathrm{c}$-Fos labeling only, the doubleimmunofluorescence procedure was separated into a 2-day sequential protocol. The rabbit anti-c-Fos primary antibody (see below) and Cy3-antirabbit secondary antibody (1:500; Jackson Immunoresearch, West Grove, PA) steps were applied over the first 24 hours, then sheep anti-TH primary antibody and Cy2 secondary antibody (1:500; Jackson) incubations were run over the subsequent 24 hours. When these labeling steps were combined as in our usual double-immunohistochemical protocol (described in Geerling et al., 2006a), a crossreaction occurred between the anti-rabbit secondary antibody and recent lots of this Chemicon sheep anti-TH antiserum.

An antiserum raised in rabbit was used to label c-Fos (1:10,000; c-Fos "Ab-5" from Oncogene; now PC38, Calbiochem, San Diego, CA). This antiserum was raised against a synthetic peptide (SGFNADYEASSSRC) corresponding to amino acids $4-17$ of human c-Fos (Calbiochem data sheet).

An additional series of rats $(n=5)$ received a small injection of an anterograde axonal tracer, Phaseolus vulgaris leucoagglutinin (PHAL), into the HSD2 subregion of the NTS. For a detailed description of PHAL injection into this region, see Geerling and Loewy (2006b). Following a 2 -day recovery, each rat underwent a 2-day period of baseline access to low-sodium chow, $\mathrm{dH} 2 \mathrm{O}$, and $3 \% \mathrm{NaCl}$. This was followed by 8-day sodium deprivation (removal of the $3 \% \mathrm{NaCl}$ drinking tube) as described above. After perfusion on day 8 at 7 AM, double-immunofluorescence labeling for c-Fos + PHAL was performed on a 1-in-5 series of transverse sections through the NTS, as well as a full series of sections through the pontine PB complex.

An antiserum raised in goat was used to label PHAL (1:20,000; \#2224 Vector, Burlingame, CA). This antiserum produces no immunoreactivity in brain tissue from cases without PHAL injections, and in each case immunoreactive axons were only found in NTS-innervated brain sites identified in previous anterograde tracing studies (Ricardo and Koh, 1978; Herbert et al., 1990; Geerling and Loewy, 2006b).

Unlike the rostrocaudally elongated NTS, nuclei in the dorsolateral pons (such as the pre-LC and the inner subdivision of PBel) span a very short rostrocaudal extent, such that, in a 1-in-5 series of sections through this region, often only one or two contain a structure of interest. Also, these samples frequently straddle the central bulk of a structure, showing only its rostral and caudal ends. To eliminate the confounding potential of such small and variable samples, c-Fos immunohistochemical staining in this region was performed on every consecutive section of tissue. This continuous series of sections extended from the level of the facial nucleus (caudal to the locus coeruleus) up through caudal levels of the inferior colliculus (roughly $25-30$ total sections per case, spanning $\approx 1.5$ $\mathrm{mm}$ ).

At the same time, a 1-in-5 series of sections through the entire brain was processed but the structures outside the NTS/AP and pontine PB complex were not analyzed in the present study. Due to the large amount of time required for histological processing, and the even greater amount of time required for subsequent image analysis, only a subset of cases could be processed at this level of detail. Thus, from the experimental groups only cases with the highest percentages of HSD2 neuronal c-Fos labeling (sodiumdeprived group, $\mathrm{n}=4$ ) and the largest volumes of $3 \% \mathrm{NaCl}$ intake ( $2 \mathrm{~h}$ saline-access group, $\mathrm{n}=5$ ) were chosen for further c-Fos immunohistochemistry and analysis in the pons and forebrain. All five control cases were stained and analyzed as well. Sodium-deprived and $2 \mathrm{~h} \mathrm{NaCl}$-access cases were processed in pairs (one case from each group per day) in order to minimize any group biases in staining intensity that could have resulted from different lengths of time in fixative prior to sectioning and staining. After image analysis (described below), select slides from each group were then counterstained with thionin (described in Geerling et al., 2006b) and re-photographed to allow sideby-side comparisons of c-Fos DAB immunohistochemistry and Nissl cytoarchitecture in the PB complex.

\section{c-Fos measurements in the NTS and AP}

As described previously (Geerling et al., 2006b), the HSD2 neurons form a compact cluster lining the medial wall of the NTS at caudalmost levels with an open fourth ventricle, and extend back through the caudal NTS in a band that parallels the border between the NTS and AP. In every case all HSD2 neurons in a 1-in-5 series of sections through the NTS were plotted and scored for the presence or absence of nuclear c-Fos immunoreactivity using conventional fluorescence microscopy and an X-Y microscope plotting system as described previously (Geerling et al., 2006a).

A total of 150-200 HSD2 neurons were counted and scored in each case. A neuron was only counted if its nucleus (devoid of HSD2, but surrounded by a rim of immunoreactivity within the cell) could be visualized within the section. All plotting was performed at $400 \times$ magnification, and a neuron was scored as c-Fos-positive only if it contained a red-fluorescent nucleus that could be clearly visualized at the same focal depth as the surrounding HSD2-ir cytoplasm. The total number of c-Fos-positive HSD2 neurons in each case was divided by the total number of HSD2 neurons, providing a percent estimate of neuronal activation.

No stereological procedure or counting correction factor was used in the present experiments, which were designed only to test for significant changes between groups after different experimental treatments, and not to produce an accurate estimate of the absolute number of labeled cells in rat brainstem. In every case the same systematic sample was taken from the same number of sections having the same thickness. The sizes of counted objects (c-Foslabeled nuclei, HSD2 neurons, and TH neurons) did not change between different treatment groups. For example, 15-20 HSD2 neurons were sampled from every case in the control, sodium-deprived, and $2 \mathrm{~h}$ saline-access groups, and their nuclear diameters (long axis) were measured. Nuclei in each group averaged 11.2 $\pm 0.2,11.5 \pm 0.2$, and $11.1 \pm 0.3 \mu \mathrm{m}$ in diameter, respectively, with no statisti- 
cally significant differences $(P=0.39$ by one-way ANOVA). When the sampling intervals, section thickness, and geometric sizes of sampled objects all remain constant, any sampling bias inherent to a cell counting method (e.g., overcounting due to the inclusion of transected objects at section edges) is similar across treatment groups, and cancels out in group-wise comparisons (Saper, 1996).

Images of HSD2 + c-Fos labeling were obtained using an Olympus FV500 laser-scanning confocal microscope. A serial Z-stack of 15-20 confocal images (step size, $1 \mu \mathrm{m}$ ) was flattened into a single 2D image using MetaMorph image analysis software (v. 6.1, Universal Imaging/ Molecular Devices, Sunnyvale, CA). Brightness and contrast adjustments were made in Adobe Photoshop (San Jose, CA), and red-channel information was duplicated into the blue channel to enhance visibility for readers with red-green color blindness.

Plotting and scoring of $\mathrm{TH}+\mathrm{c}-\mathrm{Fos}$ material were performed in an adjacent 1-in-5 series of sections, exactly as described for the HSD2 neurons above. All cases from the control group, all cases from the sodium-deprivation-only group, and all cases from the $2 \mathrm{~h}$ saline-access group were analyzed for $\mathrm{TH}+\mathrm{c}-\mathrm{Fos}$ labeling. The $1 \mathrm{~h}$ saline-access group was not analyzed due to its qualitative similarity to the $2 \mathrm{~h}$ group and the substantially greater amount of time required for $\mathrm{TH}+\mathrm{c}-\mathrm{Fos}$ plotting. All TH-ir cells were counted throughout the entire medullary section, with the exception of the dense cluster of small TH-ir neurons that fill the area postrema; the density of labeling within the AP in 50- $\mu \mathrm{m}$-thick sections precludes accurate counting (also, rarely more than a few of these cells per section contained c-Fos-immunoreactive nuclei, even in the $2 \mathrm{~h}$ saline-access group). In every section adjacent to a level containing HSD2 neurons, the A2 noradrenergic group (a cluster of roughly $400-500 \mathrm{TH}+$ neurons per case, located in the medial NTS, immediately dorsal to the dorsal motor nucleus of the vagus) was demarcated and counted using MDPlot 5.2 plotter analysis software (Accustage, Shoreview, MN). Images of $\mathrm{TH}+\mathrm{c}$-Fos labeling were obtained using conventional fluorescence microscopy, a CCD camera, and AnalySis imaging software (Soft Imaging Systems, Lakewood, CA).

Automated image analysis of nuclear c-Fos DAB immunohistochemistry was performed using the MetaMorph software package. First, in the NTS/AP of every case in this study brightfield images were captured from seven sections through the NTS (out of a 1-in-5 series, spaced $200 \mu \mathrm{m}$ apart) adjacent to those that contained HSD2 neurons (always extending from the caudalmost level with an open fourth ventricle back through a level containing the calamus scriptorius). Brightfield images of every section were captured (Nikon CCD camera and ACT-1 software) using the same light level, microscope objective $(4 \times)$, camera sensitivity, and image resolution settings $(1024 \times 1024$ pixels $)$. After all images were obtained, each was opened in MetaMorph and thresholded to an intensity range of [0-180]/256 (chosen a priori for maximum sensitivity to DAB-labeled nuclei in this material and for minimal inclusion of background noise). Next, a bilateral region of interest (ROI) containing the medial subdivision of the NTS (defined as all NTS territory medial to the solitary tract, exclusive of the dorsal vagal nucleus and area postrema; Herbert et al., 1990) was outlined manually (see the examples shown at three characteristic rostrocau- dal levels in Fig. 2A-C), and standard area and intensity counts inside this ROI were obtained using the Integrated Morphometry Analysis (IMA) feature in MetaMorph. In a minority of sections, the upper bound of the intensity threshold was reduced by between 1-9\% to prevent the counting of histological artifacts (edges or tears in the tissue, air bubbles, or slight elevations in background coloration encroaching upon the ROI). Finally, all standard area counts and intensity measurements were exported into Microsoft Excel, summed within each section, and then summed across all seven sections in each case. Counts from the area postrema were obtained similarly, using a pie wedge-shaped ROI demarcating this midline structure in three sections within the 1-in-5 series from each case (see example shown in Fig. 2B).

\section{c-Fos measurements in the pontine $\mathrm{PB}$ complex}

For measurements of c-Fos immunoreactivity in the pre-LC, a round ROI $(200 \times 200$ pixels $)$ was placed with its lateral border running along the medial curvature of the mesencephalic tract of the trigeminal nerve (see Fig. 2E). This ROI encompassed the entire cluster of c-Fos-ir that appeared in sodium-deprived rats, covering the same zone in which we previously found a heavy axonal projection from the HSD2 neurons (Geerling and Loewy, 2006b). This site, referred to as the pre-LC, lies just rostral to the locus coeruleus, dorsolateral to Barrington's nucleus, and medial to the mesencephalic nucleus of the trigeminal $(\mathrm{MeV})$. Some neurons of the $\mathrm{MeV}$ extend into the lateral part of the pre-LC and are necessarily included within this ROI, but c-Fos-ir was not seen in any of the much larger, easily recognized nuclei of these cells in any of the present experiments. Measurements inside this ROI were performed (in MetaMorph, as described above for ROIs encompassing the medial NTS and AP) in seven consecutive sections in each case (14 total images; seven each from the left and right $\mathrm{PB}$ ). These seven sections spanned $\approx 350 \mu \mathrm{m}$ through the mid-caudal PB complex (from roughly the level containing the rostralmost tip of the LC up through the caudalmost levels containing PBel), encompassing the pre-LC region of axonal input from the NTS (Geerling and Loewy, 2006b).

For measurements of c-Fos immunoreactivity in the inner subdivision of $\mathrm{PBel}(\mathrm{PBel}-\mathrm{i})$, a $300 \times 150$ pixel rectangle was positioned obliquely along the ventrolateral border of the superior cerebellar peduncle (scp; see example in Fig. 2F). This ROI was selected to encompass the majority of c-Fos labeling found in this region in 8-day sodium-deprived cases, as well as the main area of axonal input from the HSD2 subregion of the NTS (Geerling and Loewy, 2006b). Measurements inside this ROI were made in six mid-rostral sections through the PB complex (12 total images; six each from the left and right PB). These six sections spanned $\approx 300 \mu \mathrm{m}$ through the mid-rostral pontine PB complex-from the rostralmost level analyzed for pre-LC up through the caudalmost levels at which the lateral PB expands into the mesencephalic PB (where the scp becomes diffuse and medially oriented). At these same six mid-rostral levels, c-Fos-ir was also measured inside a second ROI drawn manually to include the entire lateral parabrachial nucleus ("LPBN"). As shown in Figure 2F, this ROI encompassed all the neuropil between the scp and the spinocerebellar tract, which runs along the dorsolateral border of the pons. 

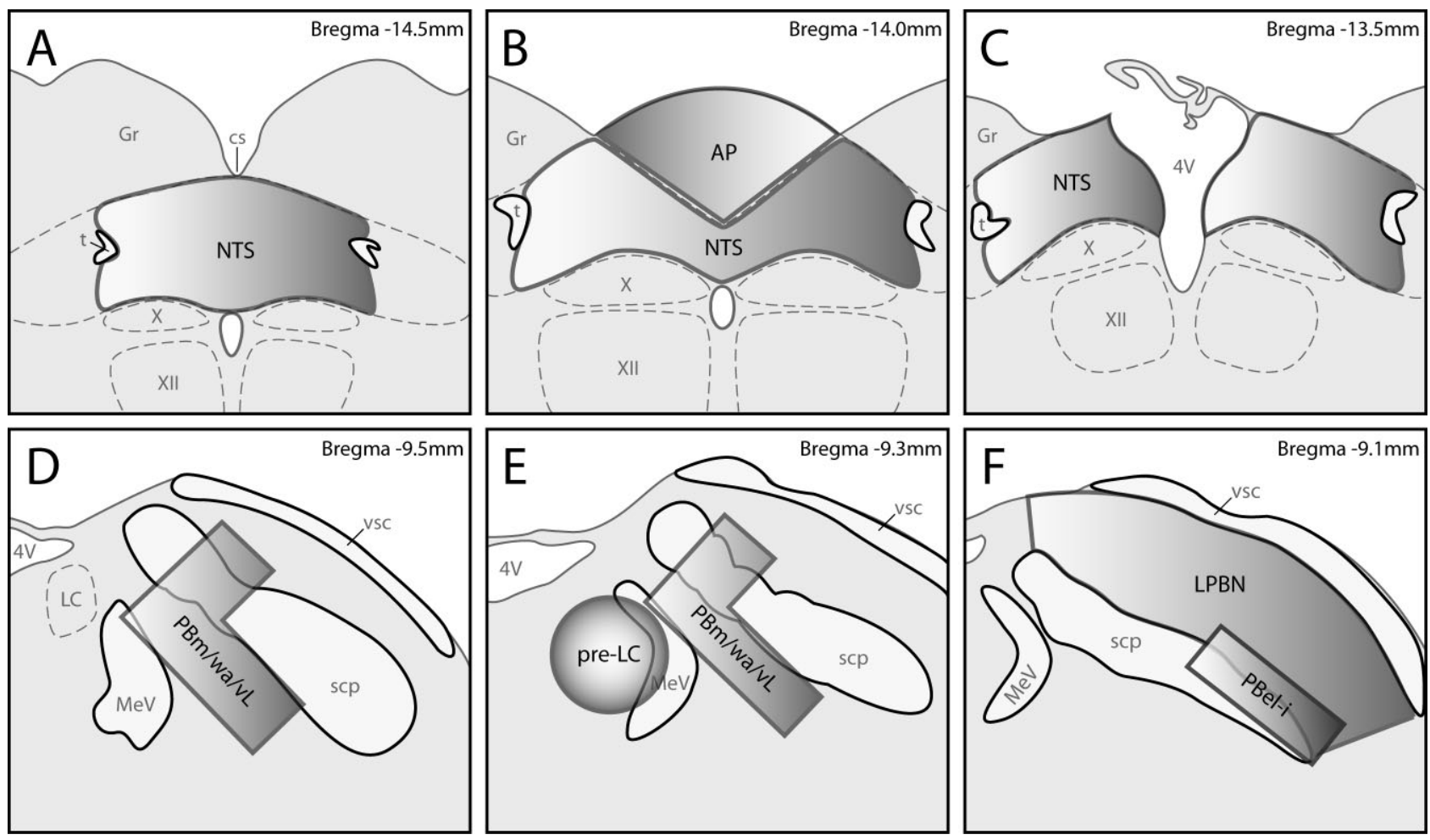

Fig. 2. These drawings show the placement of regions of interest (ROIs) used for computer measurements of c-Fos labeling. These drawings were traced over representative brain sections from this study. A-C: Three representative levels through the medial subdivision of the nucleus of the solitary tract (NTS) and area postrema (AP) in the medulla. "NTS" measurements were taken from a manually drawn bilateral ROI encompassing the medial subdivision of the NTS-defined as all NTS territory medial to the solitary tract (t) by Herbert et al. (1990). Measurements in this ROI were taken in seven sections (1-in-5 series, spaced $200 \mu \mathrm{m}$ apart) extending from the caudalmost level of the open fourth ventricle $(4 \mathrm{~V}$, shown in $\mathrm{C})$ back through the calamus scriptorius (cs). In sections that contained the AP (three sections from this 1-in-5 series), another measurement was taken in a pie wedge-shaped ROI encompassing this structure, as shown in B. D-F: Three "representative" incremental rostrocaudal levels of the pontine parabrachial nucleus (PB). In seven sections through the mid-caudal $\mathrm{PB}$ complex, measurements were taken in an inverted L-shaped ROI (shown in D-E) encompassing a large

extent of the caudal, gustatory PB nuclei-medial (PBm), waist (PBwa), and ventrolateral (PBvl). As shown in E, a circular ROI was used for measurements of c-Fos labeling in the pre-locus coeruleus (pre-LC). Measurements in this pre-LC ROI were taken in seven consecutive sections containing midlevels of the pontine $\mathrm{PB}$, placed such that the lateral edge bordered the mesencephalic nucleus and tract of the trigeminal nerve (MeV). Finally, F shows the ROIs used for measurements of c-Fos labeling in the inner subdivision of the external lateral PB nucleus (PBel-i) and for the overall lateral parabrachial nucleus (LPBN) in six consecutive sections at mid-rostral levels of the $\mathrm{PB}$ complex. Although only the right side is illustrated in $\mathrm{D}, \mathrm{E}$, note that all c-Fos measurements were taken from symmetrical ROIs on both sides of the pons. Nomenclature for the rat NTS and PB follows that of Saper and colleagues (Fulwiler and Saper, 1984; Herbert et al., 1990). Gr, gracile nucleus; scp, superior cerebellar peduncle; vsc, ventral spinocerebellar tract; $\mathrm{X}$, dorsal vagal nucleus; XII, hypoglossal nucleus.

For measurements of c-Fos-ir in gustatory regions of the $\mathrm{PB}$, an inverted, L-shaped ROI was placed at caudal and mid-caudal levels of the PB complex (see examples in Fig. $2 \mathrm{D}, \mathrm{E})$, encompassing the medial $(\mathrm{PBm})$, waist (PBwa), and so-called "ventrolateral" PB (PBvl) subnuclei as delineated by Saper and colleagues (Fulwiler and Saper, 1984; Herbert and Saper, 1990). Measurements within this ROI were taken in seven consecutive sections in each case (14 total images, seven each from the left and right $\mathrm{PB})$. These sections spanned $\approx 350 \mu \mathrm{m}$ through the caudal PB complex-from roughly the level of the mid-LC up through the mid-rostral levels analyzed for pre-LC labeling (all caudal to the sections analyzed for PBel). This rostrocaudal expanse encompassed the distinct band of intense c-Fos labeling found in caudal gustatory-recipient nuclei of the PB in all cases that were allowed to drink $3 \%$ $\mathrm{NaCl}$.

These c-Fos counts in MetaMorph (all performed using the same parameters as described for the NTS and AP) yielded the following information regarding c-Fos immunoreactivity within each predefined ROI: 1) number of immunoreactive objects, which was automatically corrected in MetaMorph by the average size of c-Fos-labeled nuclei (to prevent a blob of multiple overlapping nuclei from being counted as one object), and 2) total gray value of each object (arbitrary units), as a measure of the relative intensity of labeling in each c-Fos-immunoreactive nucleus. The numbers of c-Fos-ir objects (nuclei) measured from each ROI in each section, as well as the summed immunoreactive intensity of these objects, were summed for each case.

\section{Data analysis}

All group data are presented as mean \pm SEM. Using Prism 4 software (GraphPad, San Diego, CA), group data were analyzed by one-way ANOVA followed by Bonferroni or Tukey's post-test correction for multiple comparisons. For all comparisons involving data from MetaMorph im- 


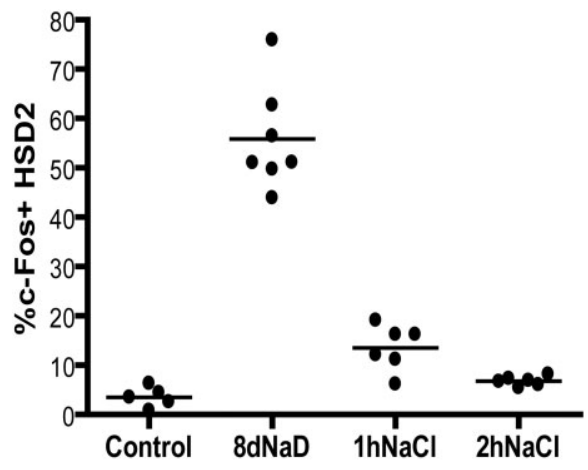

Fig. 3. Eight-day sodium deprivation significantly increased the percentage of HSD2 (11- $\beta$-hydroxysteroid dehydrogenase type 2)expressing neurons that contained nuclear immunoreactivity for the neuronal activity marker c-Fos $(P<0.001)$. This percentage was greatly reduced in 8-day sodium-deprived rats that drank $3 \% \mathrm{NaCl}$ solution (both $1 \mathrm{~h}$ and $2 \mathrm{~h} \mathrm{NaCl}$-access groups $P<0.001$ vs. sodiumdeprived; neither was significantly different from controls). $8 \mathrm{dNaD}$, 8-day sodium deprivation; $1 \mathrm{hNaCl}$, rats given access to a $3 \% \mathrm{NaCl}$ drinking tube for $1 \mathrm{~h}$ prior to perfusion; $2 \mathrm{~h} \mathrm{NaCl}$, rats given access to a $3 \% \mathrm{NaCl}$ drinking tube for $2 \mathrm{~h}$ prior to perfusion.

age analysis (c-Fos DAB labeling measurements), the total number of c-Fos-labeled nuclei, as well as their summed intensities, were analyzed separately to obtain two different measures of c-Fos activation. A significant difference in c-Fos-ir intensity (in the pre-LC) was confirmed with a nonparametric Mann-Whitney $U$-test, due to a potentially nonlinear relationship between immunohistochemical DAB labeling and actual tissue c-Fos protein quantity. Linear regression with Pearson's correlation coefficient was used for correlational analysis. For all statistical comparisons a type I error probability of $P<$ 0.05 was considered significant.

\section{RESULTS Effects of sodium deprivation and subsequent salt intake on the aldosterone- sensitive HSD2 neurons in the NTS}

Dietary sodium deprivation produced a large increase in the percentage of HSD2 neurons with c-Fos-ir nuclei. As shown in Figure 3, 55.8 $\pm 4.0 \%$ of the HSD2 neurons in sodium-deprived rats contained c-Fos-ir nuclei (range $43.9-75.9 \%, \mathrm{n}=7$ ), versus only $3.5 \pm 0.8 \%$ in nondeprived controls (range $0.8-6.3 \%, \mathrm{n}=5 ; P<0.001$ ). Examples of HSD2 and c-Fos-ir are shown in Figure 4.

Sodium deprivation also produced a large increase in sodium appetite. Rats given access to $3 \% \mathrm{NaCl}$ for 1 hour drank $7.9 \pm 1.6 \mathrm{~mL}$ (range $4-14 \mathrm{~mL}, \mathrm{n}=6$ ). In this group, only $13.5 \pm 1.9 \%$ of the HSD2 neurons still contained c-Fos-ir nuclei (range 6.2-19.1\%), representing a significant decrease versus 8-day sodium deprivation alone $(P<$ $0.001)$. Rats given $3 \% \mathrm{NaCl}$ for 2 hours drank $12.4 \pm 1.6$ $\mathrm{mL}$ (range $6-15.5 \mathrm{~mL}, \mathrm{n}=6$ ), and only $6.8 \pm 0.4 \%$ of the HSD2 neurons still contained c-Fos-ir nuclei in this group (range $5.4-8.2 \% ; P<0.001$ vs. 8 -day deprivation alone).

The time courses of $3 \% \mathrm{NaCl}$ and water ingestion by rats in the $1 \mathrm{~h}$ and $2 \mathrm{~h}$ access groups are shown in Figure 5. These two groups did not show a significant difference in saline intake at either 30 minutes or 1 hour $(P=0.12 \&$
$P=0.25$, respectively). The majority of saline intake occurred during the first 30 minutes of access $(6.4 \pm 1.4 \mathrm{~mL}$ in the $1 \mathrm{~h}$ group; $9.4 \pm 1.0 \mathrm{~mL}$ in the $2 \mathrm{~h}$ group). For comparison, the average daily intake of $3 \% \mathrm{NaCl}$ by rats in the nondeprived control group was only $0.5 \pm 0.1 \mathrm{~mL}$ (range 0-2 $\mathrm{mL}$ per 24 hours over the entire 9 -day period of measurement), and rats in the three experimental groups only ingested $0.9 \pm 0.2 \mathrm{~mL}$ of $3 \% \mathrm{NaCl}$ over the last 24 hours before removal of their saline drinking tubes (range 0-2 $\mathrm{mL}$ ).

From Figure 5B it is clear that water ingestion occurred in a pattern that was the inverse of salt ingestion, with the majority of water intake occurring after the first 30 minutes. Rats in the $1 \mathrm{~h}$ saline-access group drank $4.6 \pm 0.8$ $\mathrm{mL}$ water, and those in the $2 \mathrm{~h}$ other group drank a total of $10.4 \pm 1.4 \mathrm{~mL}$. Water intake volumes were not significantly different between these two groups at 30 minutes or 1 hour of access.

\section{Effects of sodium deprivation and subsequent salt intake on other neurons in the NTS and AP}

As shown in the photomicrograph in Figure 6A, and by the quantitative data in Figure 7, the medial subdivision of the NTS contained only light and diffuse c-Fos-ir in nondeprived control rats. Sodium deprivation produced a variable increase (particularly within the HSD2 subregion paralleling the AP-NTS border, see Fig. 6B), but this difference was not statistically significant. Salt intake, however, caused a substantial elevation in c-Fos-ir throughout the medial NTS (Fig. 6C,D). A progressive increase appeared after 1 and 2 hours of saline access, as shown in the quantitative data of Figure 7.

NTS activation in cases from these two groups showed a strong positive correlation with the amount of $3 \% \mathrm{NaCl}$ ingested. The relationship between c-Fos activation in the medial subdivision of the NTS and the ingested volume of either $3 \% \mathrm{NaCl}$ or total fluid $(\mathrm{dH} 2 \mathrm{O}+\mathrm{NaCl})$ was evaluated at two timepoints (30 minutes or 1 hour of access). The strongest overall correlation, as shown in Figure 8, was with $3 \% \mathrm{NaCl}$ intake volume (irrespective of water intake) at 30 minutes of access $(\rho=0.86, P<0.0004)$.

A portion of the c-Fos activation found in the medial NTS in each case was localized within A2 noradrenergic neurons. Like the HSD2 neurons, the A2 neurons showed a statistically significant increase in nuclear c-Fos-ir after 8 -day sodium deprivation. In control rats, $17.3 \pm 3.2 \%$ of TH-ir neurons in the medial NTS contained c-Fos-ir nuclei (range 11.6-29.4\%). After 8-day sodium deprivation this percentage increased to $35.2 \pm 2.1 \%$ (range $23.1-40.8 \%$; $P<0.01$ vs. controls, see Fig. 9). Unlike the HSD2 neurons, however, an even higher percentage of A2 neurons were activated after salt intake. After 2 hours access to $\mathrm{NaCl}$, c-Fos-ir nuclei were found within $46.8 \pm 3.4 \%$ of the TH-ir neurons in the medial NTS (range 38.7-62.0\%; $P<$ 0.05 vs. sodium deprivation). Across all cases from the control, 8-day sodium-deprived, and $2 \mathrm{~h}$ NaCl-access groups, the percentage of c-Fos-labeled A2 neurons showed a positive linear correlation with the number of c-Fos-labeled nuclei in the medial NTS $(\rho=0.74 ; P=$ 0.005; see Fig. 10). No significant correlation between c-Fos in A2 neurons versus HSD2 neurons was found within or across groups. Figure 11 shows an example of nuclear c-Fos immunoreactivity in the A2 neurons of a 

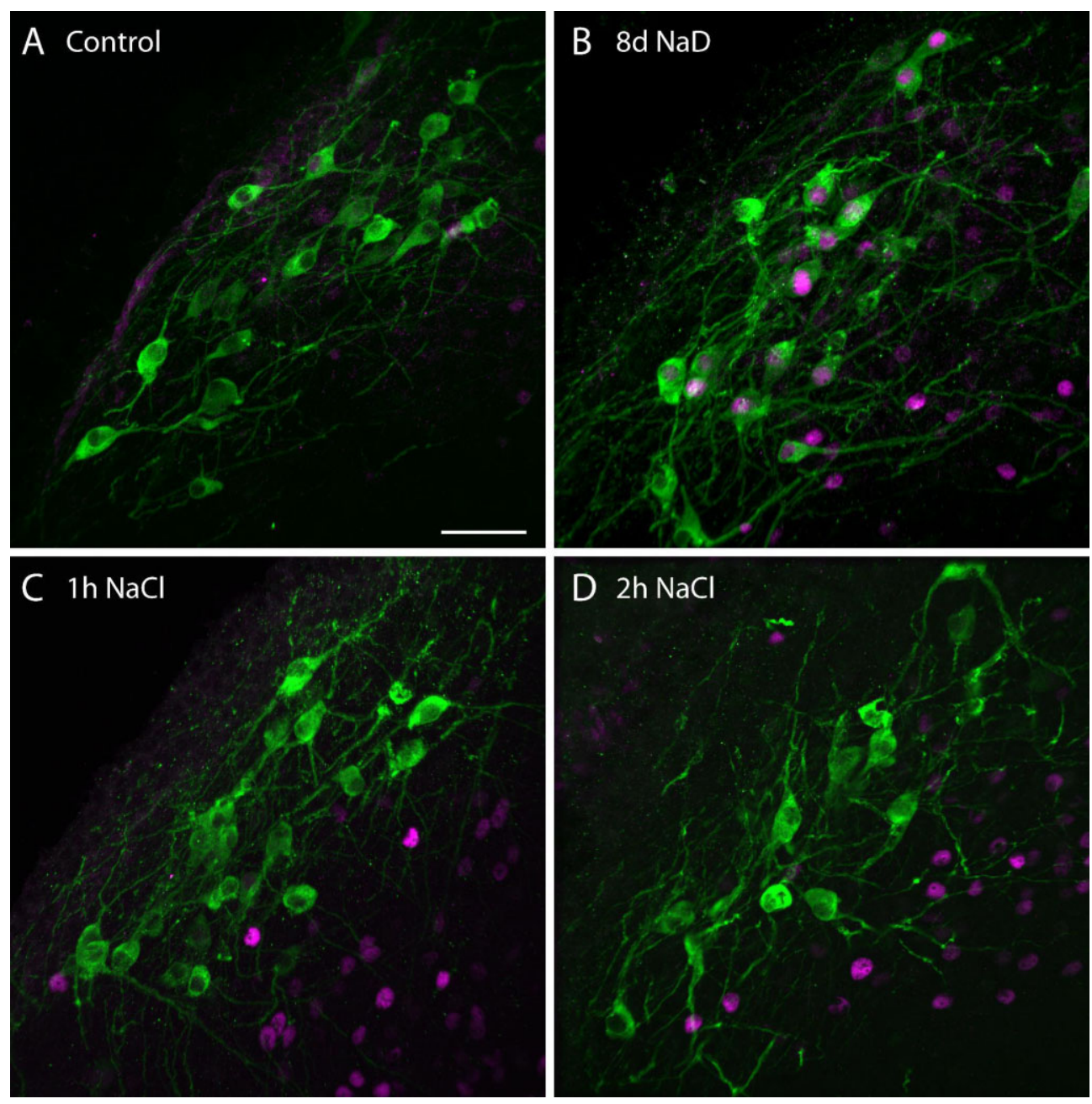

Fig. 4. A: In the nucleus of the solitary tract (NTS) of control cases, very few HSD2 neurons (green) contained the neuronal activity marker c-Fos (magenta). B: The HSD2 neurons were, however, markedly activated by sodium deprivation $(8 \mathrm{dNaD})$, as evidenced by their

case from the $2 \mathrm{~h}$ saline-access group (note that, of the many c-Fos-labeled nuclei in the medial NTS, only a minority were found within TH-ir neurons).

In the AP, changes in c-Fos activation generally paralleled those measured in the underlying NTS. As can be seen in Figure 6, c-Fos activation in the AP was never more prominent than in the medial NTS. The increased c-Fos labeling of rats in the sodium-deprived and $1 \mathrm{~h}$ saline-access groups were not statistically significant; in the $2 \mathrm{~h}$ saline-access group, however, the number of labeled nuclei was significantly increased versus controls $(P<0.05$; see Fig. 12). Across all cases from all groups, the number of c-Fos-ir nuclei in the AP showed a positive linear correlation with that measured in the underlying NTS ( $\rho=0.81 ; P<0.0001$; see Fig. 13 ). Also, just like c-Fos activation in the underlying NTS, c-Fos activation in the AP among cases in both saline-access groups correlated positively with the amount of $3 \% \mathrm{NaCl}$ ingested at 30 minutes of access ( $\rho=0.78 ; P=0.003$; see Fig. 14). increased nuclear c-Fos immunoreactivity. C,D: This activation was greatly reduced just 1-2 hours after voluntary ingestion of $3 \% \mathrm{NaCl}$. Scale bar $=50 \mu \mathrm{m}$ in A (applies to all).

\section{Sodium deprivation activates pre-LC and PBel-i}

In control cases, only light and scattered c-Fos labeling was found throughout most of the PB complex and surrounding pons, as shown in Figure 15. One exception was a small group of intensely labeled nuclei found in every case at mid-rostral levels of the PB complex (described below, see Fig. 15C).

In every 8-day sodium-deprived case, the most salient feature in the dorsolateral pons was a dense cluster of c-Fos-immunoreactive nuclei found bilaterally at midcaudal levels of the PB complex, centered just medial to the mesencephalic nucleus of the trigeminal nerve $(\mathrm{MeV})$, as shown in Figure 16B. This site, which receives a major axonal projection from the HSD2 neurons, lies immediately rostral to the locus coeruleus (LC), so we refer to it as "pre-LC" (Geerling and Loewy, 2006b).

Another consistent feature in sodium-deprived cases was a loose cluster of c-Fos labeling found bilaterally at 


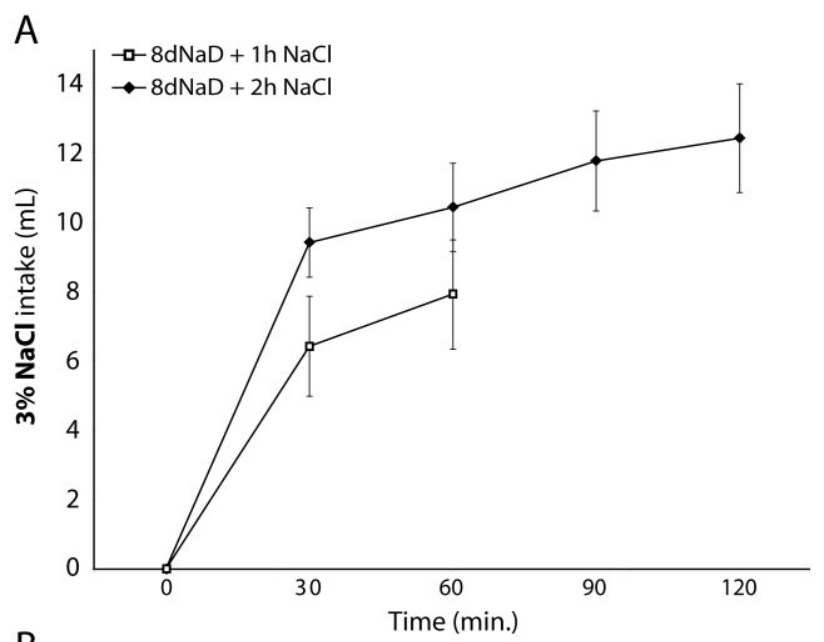

B

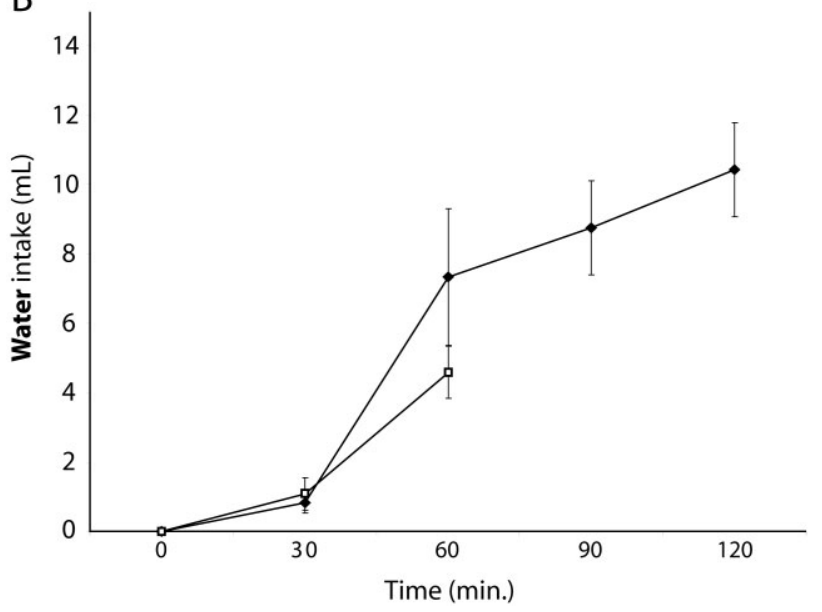

Fig. 5. Average volumes of $(\mathbf{A}) 3 \% \mathrm{NaCl}$ and (B) water consumed by rats in the $1 \mathrm{~h}$ and $2 \mathrm{~h} \mathrm{NaCl}$-access groups. Note the inverse relationship between the rapid ingestion of saline and the delayed (and relatively small) water intake. Group differences were not statistically significant for either fluid at any time.

mid-rostral levels of the PB complex, bordering and penetrating into the superior cerebellar peduncle along its ventrolateral edge (see Fig. 16C). These c-Fos-ir nuclei, somewhat less numerous than those found in the pre-LC, were primarily located within the inner subdivision of the PBel-i, which also receives axonal input from the HSD2 neurons (Geerling and Loewy, 2006b).

Marked activation in these two sites-pre-LC and PBel-i-was observed in every sodium-deprived case. Other subnuclei in the PB complex contained only occasional c-Fos labeling, similar to controls. As shown in Figure 18, c-Fos activation in the pre-LC after 8-day sodium deprivation represented a statistically significant increase over control levels $(P<0.001)$. The $2 \mathrm{~h}$ salineaccess group showed a tendency toward fewer c-Fos-ir nuclei in the pre-LC, relative to 8-day sodium deprivation. This decrease was not statistically significant $(P>0.05)$, although a reduction in total immunoreactive intensity of c-Fos labeling in these cases represented a statistically significant decrease versus the sodium-deprived group $(P<0.05)$. This decrease was confirmed by a nonparamet- ric statistical test because the immunoreactive intensity measured after DAB enzymatic labeling may have a nonlinear relationship with actual c-Fos protein levels $(P=$ 0.03 by one-tailed Mann-Whitney $U)$; this statistically significant difference in intensity without a similarly significant decrease in c-Fos number was the only such example in the present experiments. Despite this apparent reduction in c-Fos-ir intensity, labeling in the $2 \mathrm{~h}$ salineaccess group remained significantly elevated relative to controls $(P<0.01$ and $P<0.05$, measured as the number or intensity of labeling, respectively).

c-Fos activation in the PBel-i was not as large as that measured in the pre-LC. Although it may not seem apparent from the quantitative data shown in Figure 19, c-Fos labeling in this site was a conspicuous feature in all sodium-deprived cases due to the relatively low level of c-Fos labeling in the surrounding lateral PB (see Fig. 16B). As in the pre-LC, the increased number of c-Foslabeled nuclei in the PBel-i produced by 8-day sodium deprivation was statistically significant $(P<0.05$ vs. controls). Activation in the PBel-i was less conspicuous in $2 \mathrm{~h}$ saline-access cases (due to a large increase in surrounding c-Fos activation in the LPBN, described below), but it was sustained or even increased relative to 8-day sodiumdeprived cases, in contrast to the decreased intensity found in the pre-LC. In $2 \mathrm{~h}$ saline-access cases, neither the number nor the intensity of labeling were significantly different from sodium-deprived cases, and both remained significantly elevated versus controls $(P<0.01, P<0.05$, respectively).

\section{Axons from the HSD2 subregion of the NTS envelop the c-Fos-activated regions of the pre-LC and PBel-i after sodium deprivation}

Additional experiments were conducted in order to compare the locations of c-Fos labeling in the PB complex after 8-day sodium deprivation with the distribution of axons from the HSD2 subregion of the NTS (Geerling and Loewy, 2006b). From an additional series of 8-day sodiumdeprived rats that received PHAL injections into the NTS $(\mathrm{n}=5)$, two cases contained small injection sites centered within the distribution of the HSD2 neurons in the NTS. The injection site from one of these two cases (\#8616) is shown in Figure 20.

Similar to the 8-day sodium-deprived cases described above, these cases exhibited substantial activation of the HSD2 neurons in the NTS. In case 8616 , for example, $62 \%$ (116 of 186 HSD2 neurons) contained c-Fos-ir nuclei. Also as before, a prominent cluster of c-Fos-ir nuclei was found bilaterally in the pre-LC of each case. As shown in Figure $21 \mathrm{~A}$, c-Fos labeling in the ipsilateral pre-LC was enmeshed in a dense terminal field of PHAL-labeled axons from the NTS. An identical cluster of c-Fos labeling was found in the pre-LC contralateral to the injection site (not shown), amid a reduced density of PHAL-labeled axons in the same distribution.

As described above, many c-Fos-ir nuclei were also found bilaterally within the PBel-i. Most of these nuclei were situated along the ventrolateral edge of the superior cerebellar peduncle, and others formed streams penetrating into the peduncle. As shown in Figure 21B, c-Fosactivated neurons in the PBel-i ipsilateral to the injection site were surrounded by PHAL-labeled axons from the NTS, many of which cascaded into the peduncle in a 

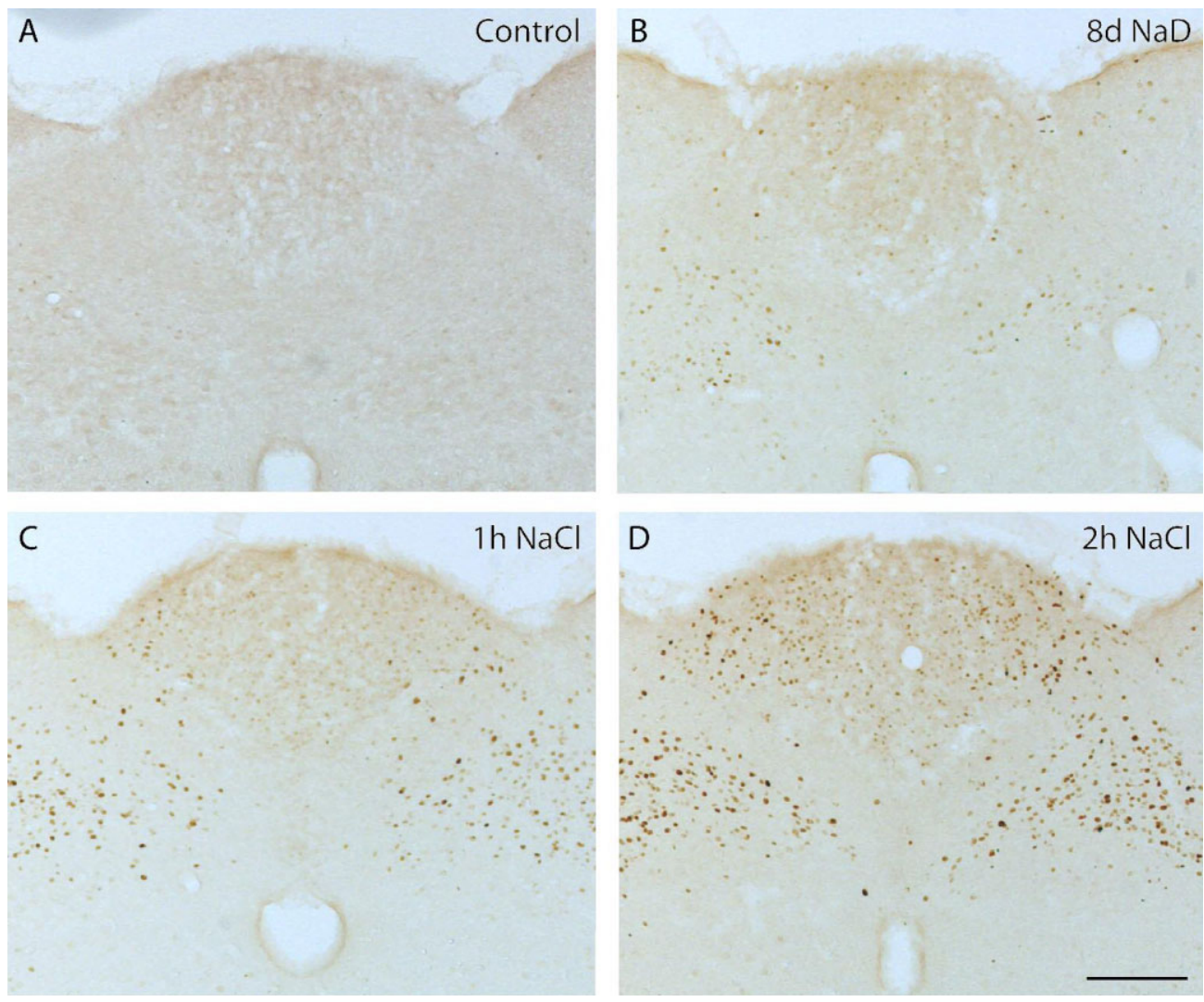

Fig. 6. A: In control cases, c-Fos labeling was light and diffuse within the medial subdivision of the NTS (defined as all NTS neurons medial to the solitary tract, see Herbert et al., 1990) and the area postrema (AP). B: Eight-day dietary sodium deprivation $(8 \mathrm{dNaD})$ produced a mild and variable increase in some cases, but the overall group difference was not statistically significant versus controls (note, however, the clusters of c-Fos labeling in the HSD2 subregion of the NTS ventrolateral to the AP, and the scattered c-Fos labeling located further laterally within the medial NTS, in the region containing A2

noradrenergic neurons). C,D: Ingestion of concentrated saline (1h and $2 \mathrm{~h} \mathrm{NaCl}$ groups) produced a dramatic and statistically significant increase in c-Fos activation throughout much of the medial NTS as well as the AP (particularly in its lateral extent). In each case from these two groups, neighboring levels through the caudal two-thirds of the medial NTS (not shown) contained a similar pattern of c-Fos labeling, spanning from where this nucleus borders the caudalmost open fourth ventricle rostrally back through levels containing the calamus scriptorius caudally. Scale bar $=200 \mu \mathrm{m}$ in D (applies to all).

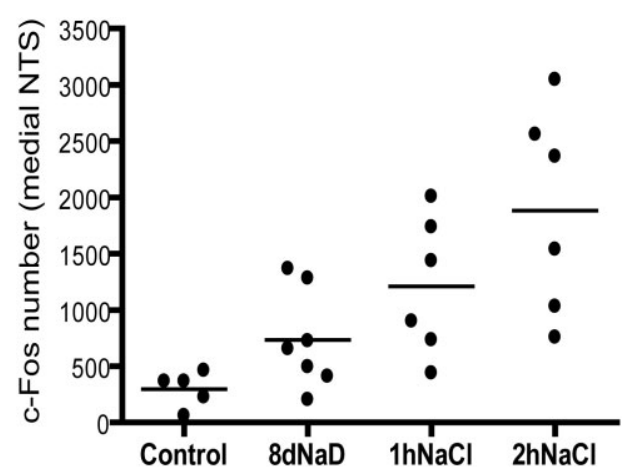

Fig. 7. Total neuronal activation in the medial NTS was markedly elevated after the ingestion of concentrated saline. The $2 \mathrm{~h}$ salineaccess group showed statistically significant increases in the number of c-Fos-ir nuclei versus both controls $(P<0.01)$ and sodium-deprived rats $(P<0.05)$. No other group differences were statistically significant, despite the increasing trend across the control, $8 \mathrm{~d} \mathrm{NaD}$, and $1 \mathrm{~h}$ $\mathrm{NaCl}$ groups.

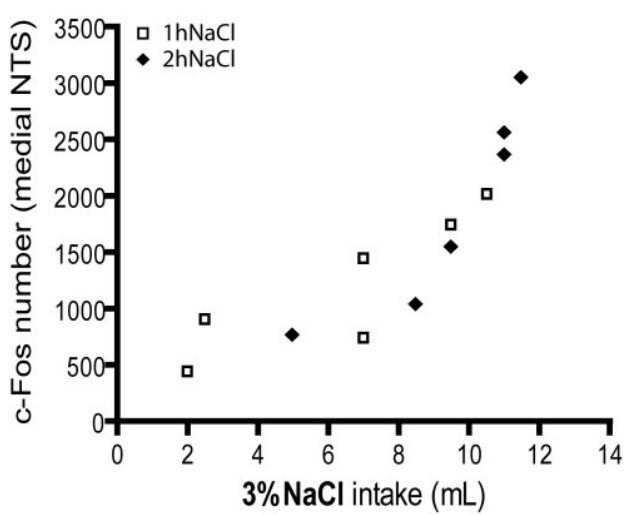

Fig. 8. Neuronal c-Fos activation in the medial NTS correlated positively with the amount of $3 \% \mathrm{NaCl}$ ingested by 30 minutes of access to saline in cases from the $1 \mathrm{hNaCl}$ and $2 \mathrm{hNaCl}$ groups $(\rho=$ $0.86, P=0.0004)$. 


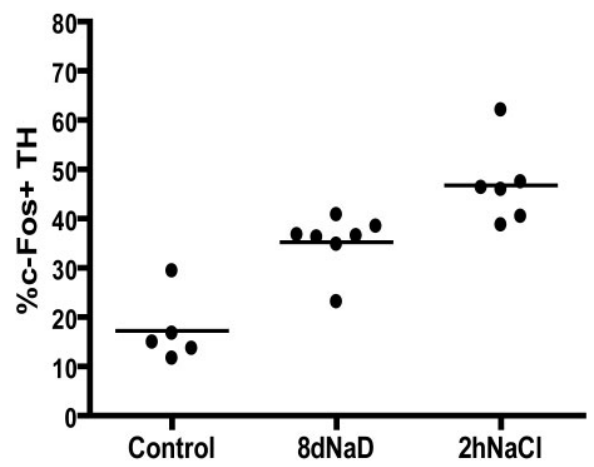

Fig. 9. The percentage of tyrosine hydroxylase (TH)-immunoreactive neurons in the medial NTS (the A2 noradrenergic group) containing nuclear c-Fos immunoreactivity increased significantly during 8-day sodium deprivation $(P<0.01)$. In contrast to the inactivation of neighboring HSD2 neurons, the percentage of c-Fos-activated A2 neurons increased even further after salt intake $(P<0.001$ vs. controls; $P<0.05$ vs. sodium-deprivation).

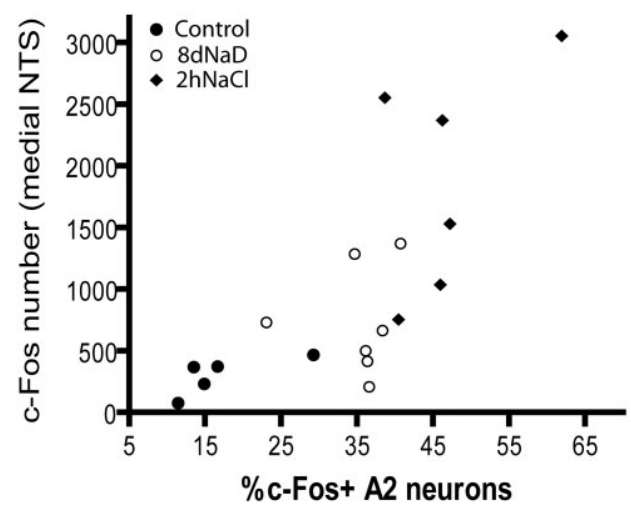

Fig. 10. Activation of TH-labeled catecholaminergic neurons in the A2 noradrenergic group, unlike the HSD2 neurons, correlated with the overall activation of neurons in the surrounding medial NTS. The percentage of c-Fos-activated neurons in the A2 group showed a significant positive correlation with the overall number of c-Foslabeled nuclei in the medial NTS $(\rho=0.74, P=0.0005)$ across al cases in which TH $+\mathrm{c}$-Fos was analyzed $(\mathrm{n}=18$, all cases from the control, 8-day sodium-deprived, and $2 \mathrm{~h}-\mathrm{NaCl}$ groups).

similar pattern. As in the pre-LC, an identical pattern of c-Fos labeling was found in the contralateral PBel-i (not shown), amid a reduced density of axonal labeling in a similar pattern.

\section{Increased c-Fos within the overall LPBN after salt intake}

The lateral parabrachial nucleus, or "LPBN," encompasses a number of nuclei lying between the superior cerebellar peduncle and the dorsolateral pontine surface (Fulwiler and Saper, 1984). The LPBN has been implicated in the negative feedback control of salt and water intake in various pharmacological experiments (Menani et al., 1996, 1998; Johnson and Thunhorst, 1997; Menani and Johnson, 1998). Primarily for comparison with these prior physiologic studies (and also because the boundaries of most PB nuclei in this region could not be unambiguously determined in many of the c-Fos-labeled sections

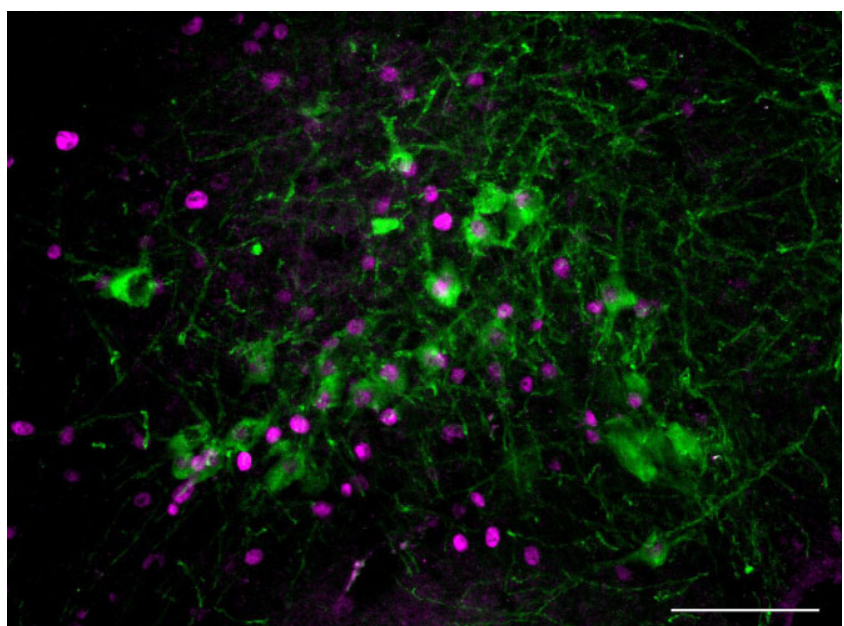

Fig. 11. This photomicrograph, taken from a $2 \mathrm{~h}$ saline-access case (\#8068) shows nuclear c-Fos labeling (magenta) within and surrounding many of the A2 noradrenergic neurons in the medial NTS (green, TH-immunoreactivity). Note that while c-Fos activation was found in a substantial proportion of the A2 neurons, most c-Fos labeled nuclei in the medial NTS were not localized within TH-ir neurons. In fact, as is evident in this figure, the nuclei with stronger c-Fosimmunoreactivity tended to be found outside the A2 group. Scale bar $=100 \mu \mathrm{m}$

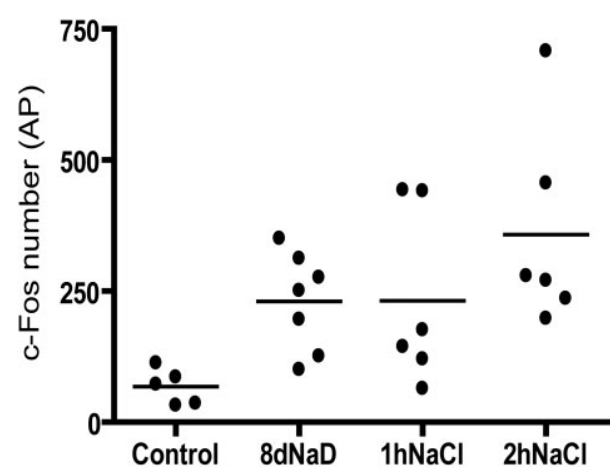

Fig. 12. The increase in nuclear c-Fos labeling within the AP was statistically significant in $2 \mathrm{~h}$ saline-access cases, relative to controls $(P<0.05)$. No other group differences were statistically significant, due to the inherently smaller sample size in the AP and large variations in c-Fos labeling in each group.

from the present experiments), c-Fos labeling was quantified in the overall LPBN throughout mid-rostral levels of the pontine PB (see Materials and Methods).

Consistent with prior data implicating this region in post-ingestive negative feedback signaling, the number of c-Fos labeled nuclei in the LPBN showed a statistically significant increase after salt ingestion $(P<0.05,2 \mathrm{~h} \mathrm{NaCl}$ vs. controls; see Fig. 22). In cases from the $2 \mathrm{~h}$ saline-access group, a widespread pattern of activation appeared throughout much of the LPBN. Amid this widespread increase in c-Fos labeling, somewhat more conspicuous concentrations of c-Fos labeling were seen in regions corresponding to the locations of the rostral PBdL and outer subdivision of PBeL (see example in Fig. 17C).

Sodium-deprived rats showed a nonsignificant tendency toward increased c-Fos labeling in the LPBN relative to 


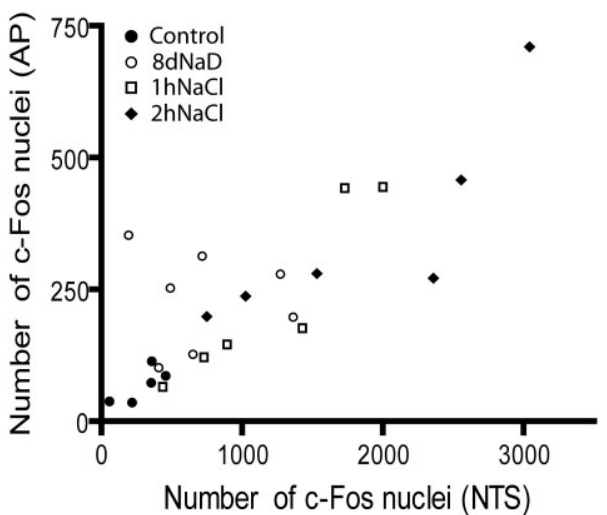

Fig. 13. In the AP, c-Fos activation correlated positively with that in the underlying NTS $(\rho=0.81, P=0.0001)$.

the control group (due to the consistent group of c-Foslabeled nuclei in PBel-i). The statistical significance of this difference may have been obscured by an unexpected group of c-Fos-labeled nuclei found in control rats. As shown in Figure 15C, every case in the control group $(\mathrm{n}=$ 5) contained a small but prominent group of c-Fos labeling within the LPBN, found in a position roughly corresponding to a dorsolateral region of the central lateral nucleus (PBcl). The bulk of this cluster spanned $\approx 4-7$ sections (each 50- $\mu \mathrm{m}$-thick, $\approx 200-350 \mu \mathrm{m}$ in total length) through the mid-rostral pontine PB complex.

\section{Salt intake increases c-Fos in caudal PB}

Clearly, the greatest c-Fos activation throughout the greatest extent of the PB was found in animals that had ingested concentrated saline. Despite this substantial activation in the mid-rostral LPBN, however, the most intense nuclear c-Fos labeling found in saline-access cases was located in the caudal, gustatory region of the $\mathrm{PB}$, as shown in Figure 17A. An analysis of gustatory relay nuclei in the caudal PB was not a part of our original experimental design, but the consistent appearance of intense c-Fos labeling here after salt intake prompted us to perform additional measurements in a zone encompassing the medial (PBm), waist (PBwa), and so-called "ventrolateral" (PBvl) subnuclei at caudal levels of the PB complex (see Materials and Methods for details; naming conventions follow Fulwiler and Saper, 1984). From the caudalmost levels of the PBm (coexistent with the rostral locus coeruleus) up to roughly mid-caudal levels of the pre-LC, this inverted-L-shaped stripe of gustatory relay nuclei exhibited intense c-Fos labeling after salt ingestion (see Fig. 17A). Every rat from the $2 \mathrm{~h} \mathrm{NaCl}$-access group exhibited this pattern of intense c-Fos activation, whereas control and sodium-deprived cases contained very little labeling in this region (quantitative data shown in Fig. 23).

\section{Lack of c-Fos activation in BSTvl during sodium deprivation}

Although the analysis of brain regions outside the NTS/AP and PB complex was also not originally within the scope of this study, one unexpected preliminary finding will be mentioned here for the purpose of comparison. The fusiform subnucleus of the ventrolateral bed nucleus of the stria terminalis (BSTvl), which receives substantial

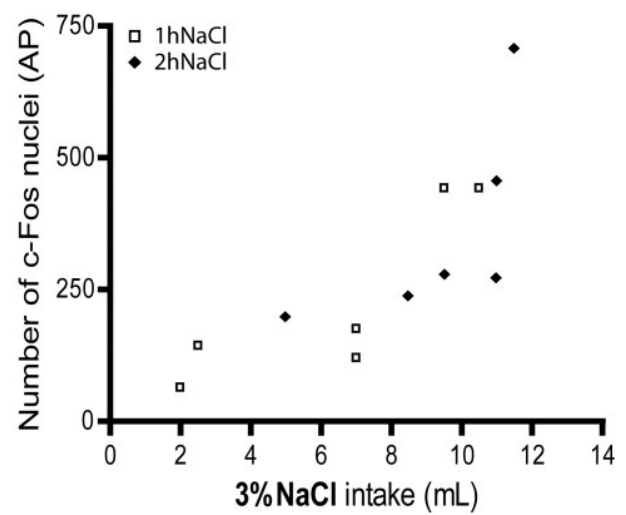

Fig. 14. Similar to the correlation shown in Figure 8 for the NTS, salt intake and c-Fos activation in the AP correlated positively. This correlation is shown as the volume of $3 \% \mathrm{NaCl}$ ingested at 30 minutes (by rats from the $1 \mathrm{~h}$ and $2 \mathrm{~h}$ saline-access groups) versus the number of c-Fos-ir nuclei measured within the AP $(\rho=0.78, P=0.003)$.

axonal input from the HSD2 neurons and from neighboring A2 noradrenergic neurons in the NTS (Geerling and Loewy, 2006b), did not exhibit any increase in c-Fos immunoreactivity after 8-day sodium deprivation.

Based on the heavy axonal projection from HSD2 neurons to the BSTvl, similar in magnitude to the pre-LC, we had expected a similar increase c-Fos labeling in this site. Surprisingly, however, none of the cases in this study (including cases in the control and $2 \mathrm{~h}$ saline-access groups) contained more than a few scattered c-Fos-labeled nuclei in or around this site. Figure 24 shows an example of the relative lack of c-Fos activation after 8-day sodium deprivation in this subregion of BSTvl, which is delineated by its dense axonal input from the HSD2 subregion of the NTS (anterograde PHAL labeling from a sodium-deprived, PHAL-injected case, \#8616, described above).

\section{DISCUSSION}

The two most important findings of the present study are that 1) while the HSD2 neurons in the NTS are acti-

Fig. 15. A-C: c-Fos immunoreactivity in a representative case from the control group (\#8134) at three rostrocaudal levels of the dorsolateral pons. Adjacent panels $\left(\mathrm{A}^{\prime}-\mathrm{C}^{\prime}\right)$ show the same three sections counterstained with thionin to reveal local cytoarchitecture. Overall, the dorsolateral pons contained very little c-Fos labeling in control cases. A: At caudal levels of the pontine parabrachial complex (PB), gustatory relay neurons form a band through the medial (PBm), waist (PBwa), and ventrolateral ( $\mathrm{PBvl}$ ) subnuclei. These sections also contain the rostral extent of the locus coeruleus (LC). B: Mid-levels of the $\mathrm{PB}$ contain the central bulk of the pre-locus coeruleus (pre-LC), as well as the rostralmost extents of the gustatory relay nuclei centered in the caudal PB. C: Mid-rostral levels of the pontine LPBN contain the bulk of NTS-innervated nuclei, including the inner and outer subdivisions of the external lateral nucleus (PBel), the central lateral nucleus (PBcl), and the dorsal lateral nucleus (PBdl; nomenclature from Fulwiler and Saper, 1984; Herbert et al., 1990). Note the dense cluster of c-Fos-labeled nuclei in the dorsolateral PBcl. Despite the relative paucity of c-Fos in the rest of the lateral $\mathrm{PB}$, this distinct cluster c-Fos-labeled nuclei was found in every control case. MeV, mesencephalic nucleus of the trigeminal nerve; scp, superior cerebellar peduncle. Scale bar $=200 \mu \mathrm{m}$ in A (applies to all). 
A

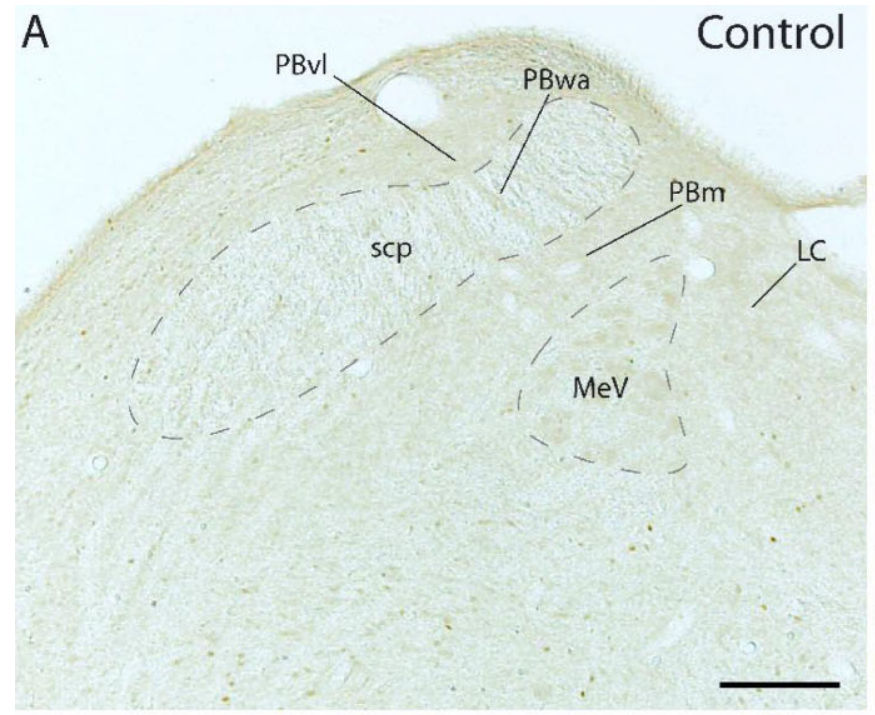

B

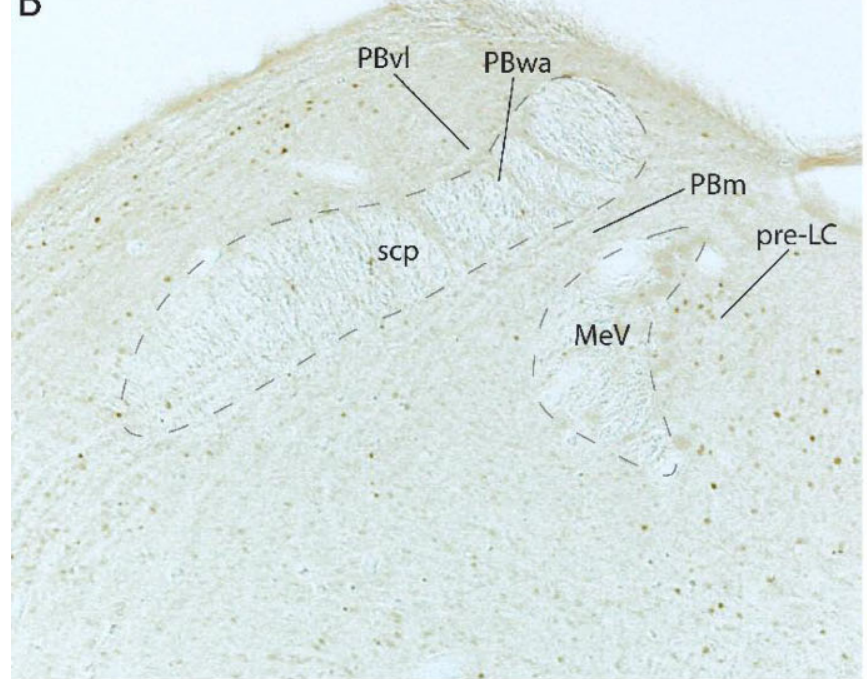

C

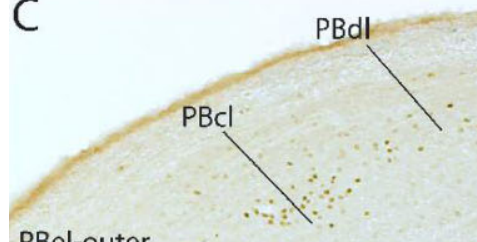

PBel-outer

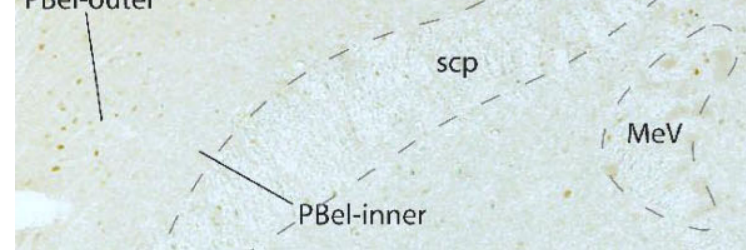

\section{$A^{\prime}$}

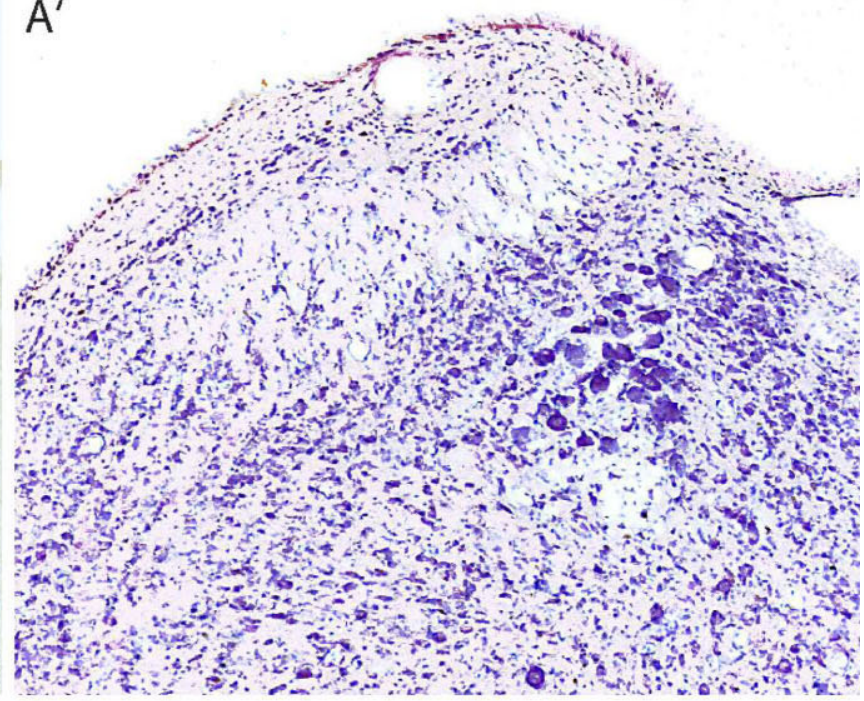

$\mathrm{B}^{\prime}$

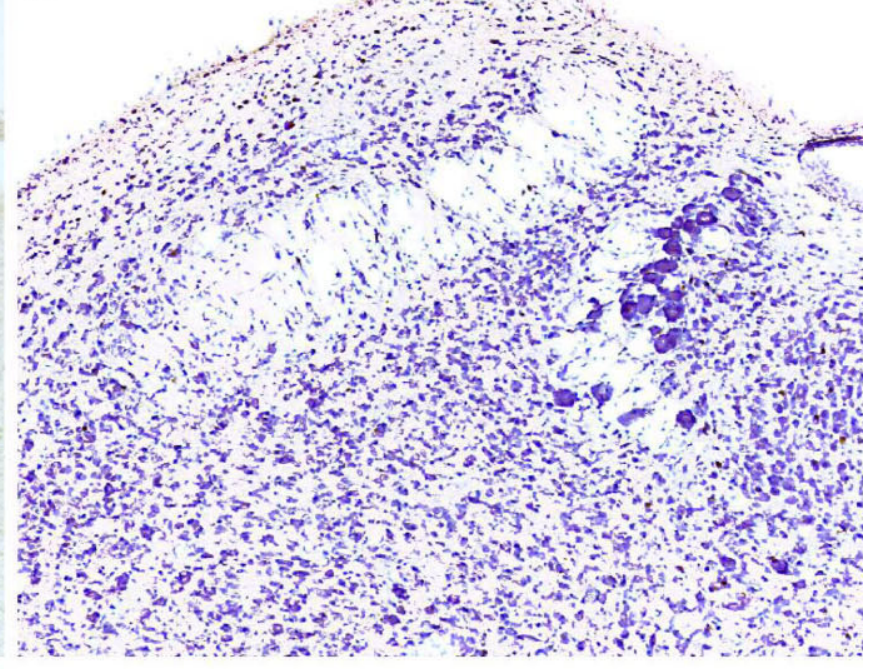

$C^{\prime}$

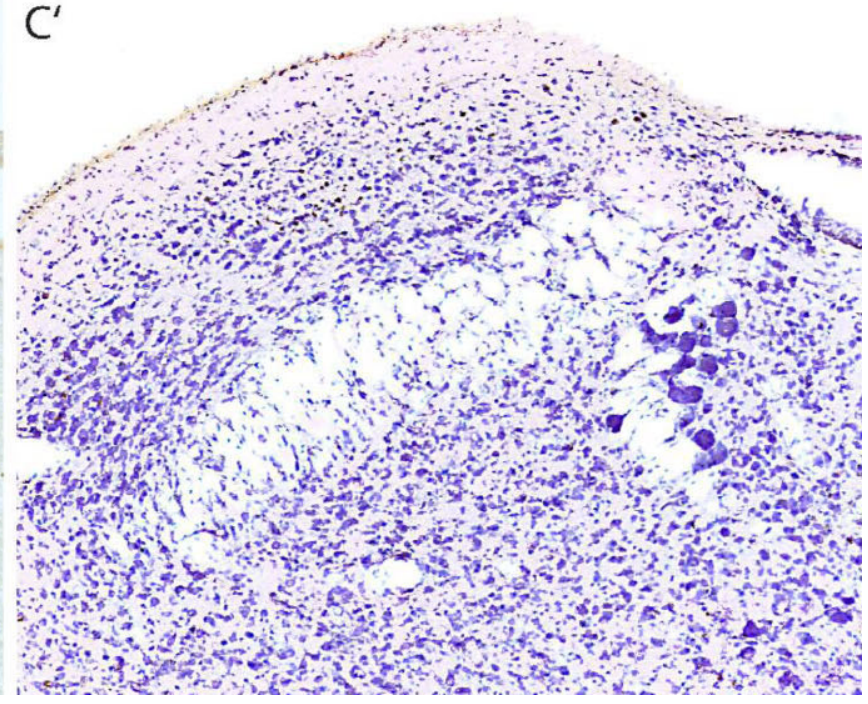




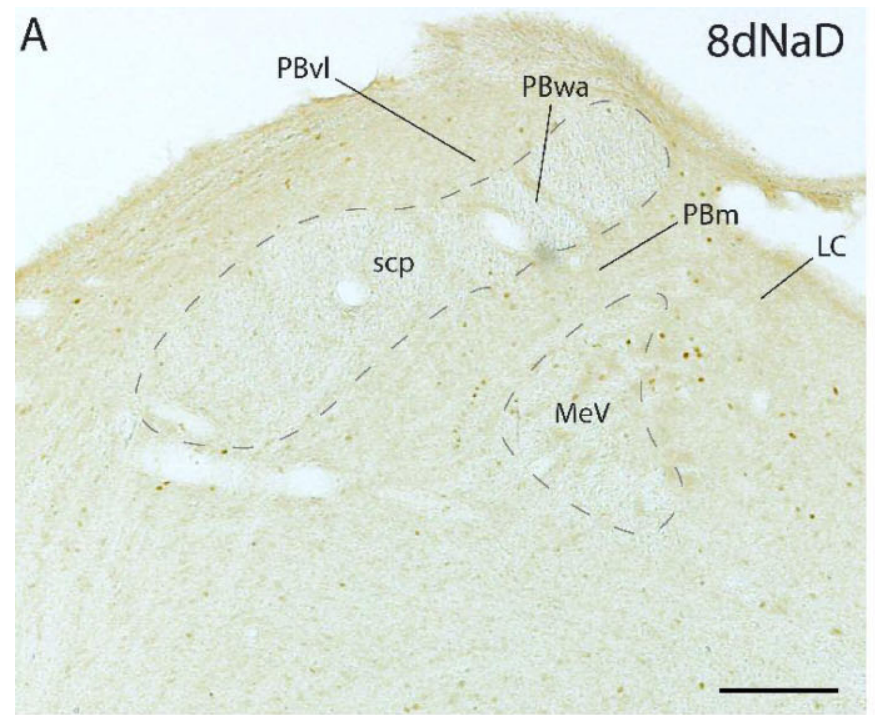

B

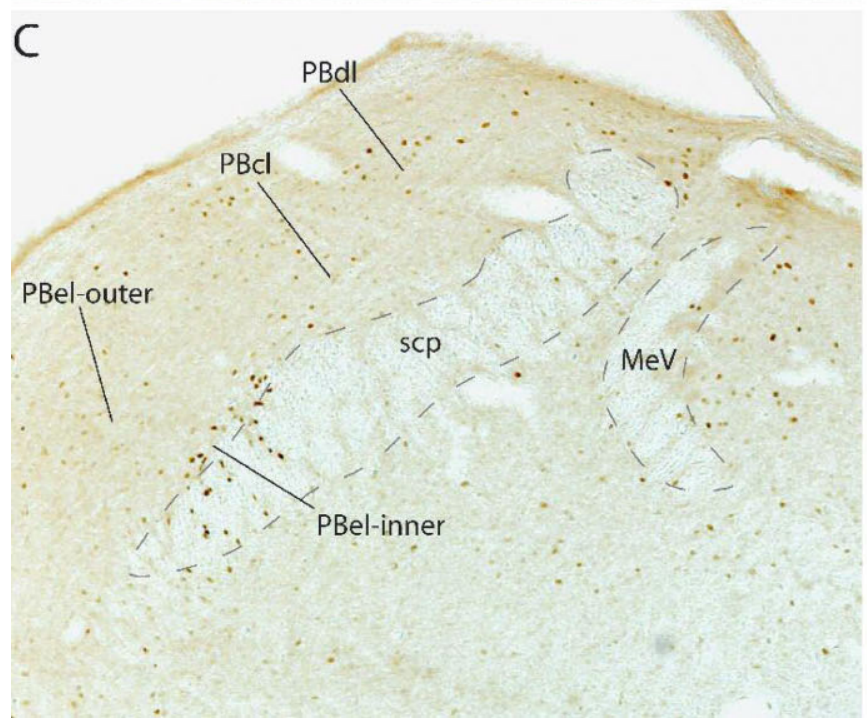

Fig. 16. A-C: c-Fos immunoreactivity at three rostrocaudal levels of the dorsolateral pons in a representative case from the 8-day sodium-deprived group ( $8 \mathrm{dNaD}$, \#8057). Adjacent panels $\left(\mathrm{A}^{\prime}-\mathrm{C}^{\prime}\right)$ show the same three sections counterstained with thionin to reveal local cytoarchitecture. These three incremental rostrocaudal levels of the PB complex roughly correspond to those described above for Figure 15. Note the two prominent clusters of c-Fos-labeled nuclei in: 1) the

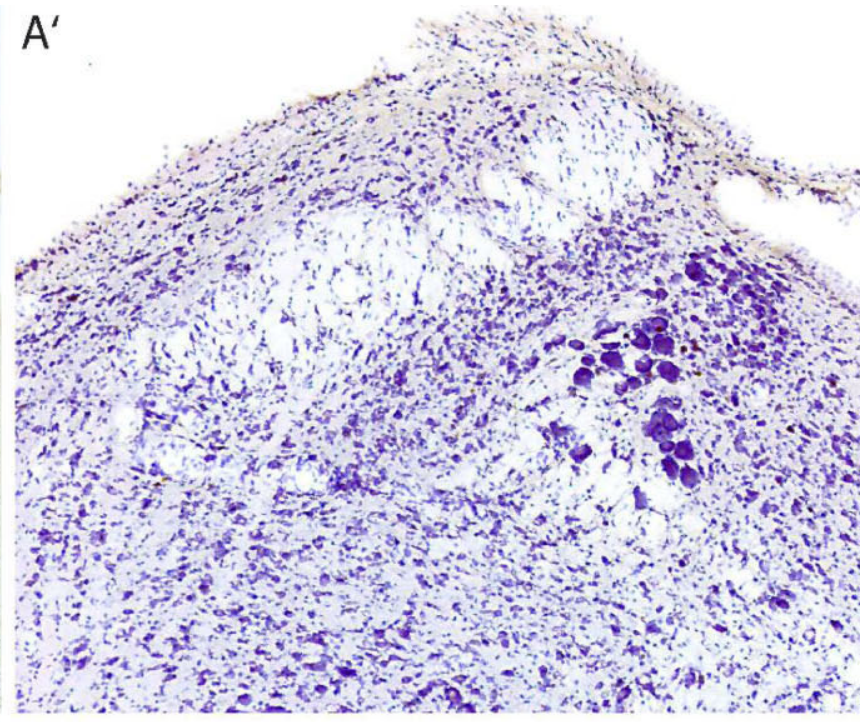

$\mathrm{B}^{\prime}$
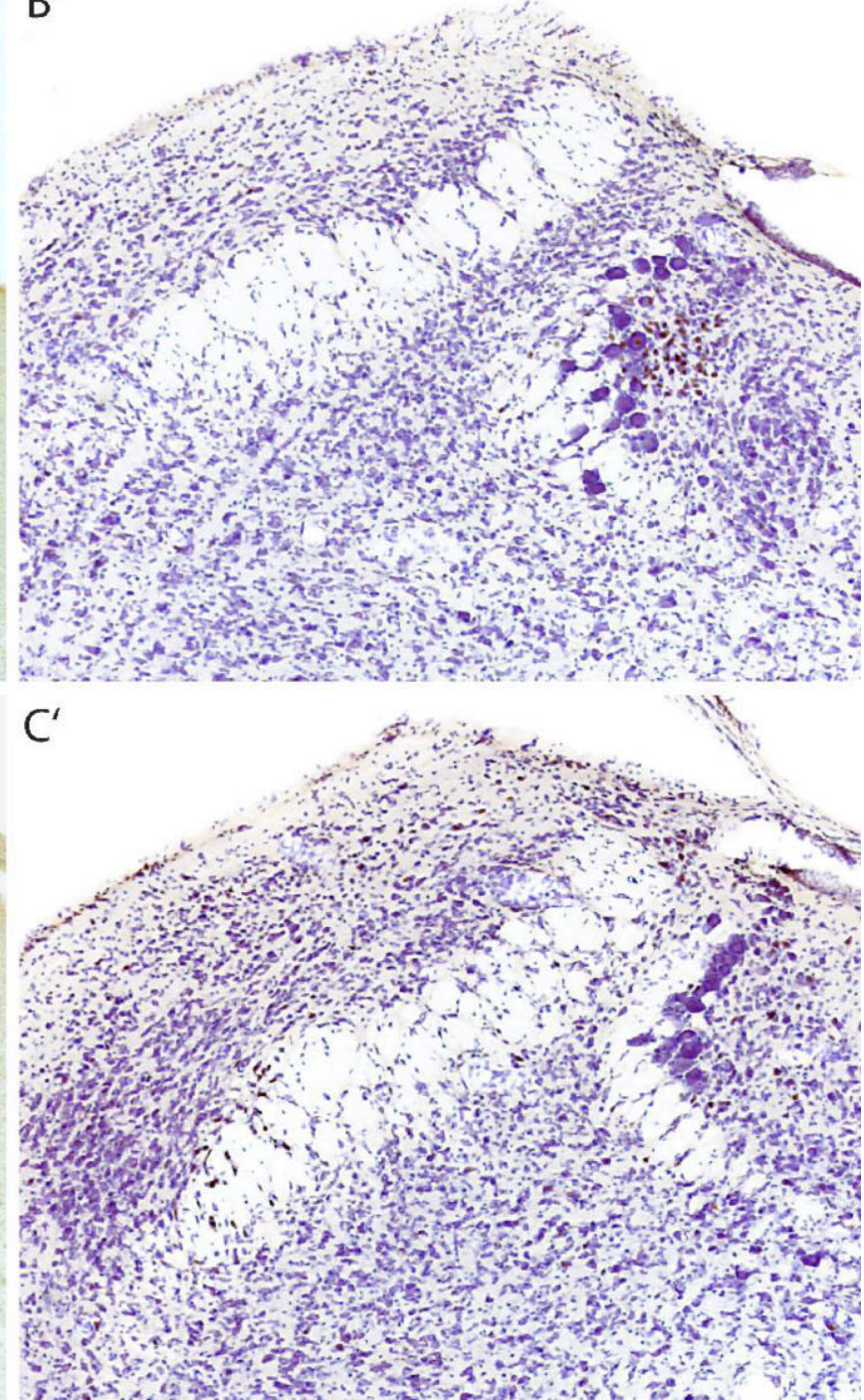

pre-LC at mid-levels of the $\mathrm{PB}$ complex (shown in B) and 2) the innermost part of PBel-i at mid-rostral levels of the PB complex (bordering and penetrating into the scp in C). These two clusters of c-Fos labeling were found in every sodium-deprived case. Other PB nuclei contained little or no c-Fos labeling, similar to the light and scattered labeling found in control cases. Bar, Barrington's nucleus. Scale bar $=200 \mu \mathrm{m}$ in A (applies to all). 
vated specifically by chronic sodium deficiency, an even larger number of other neurons in the NTS and AP are activated following salt intake, and 2) neurons in the pre-LC and $\mathrm{PBel}-\mathrm{i}-$ pontine sites that receive direct axonal input from the HSD2 neurons-are activated in concert with the HSD2 neurons during chronic sodium deficiency, whereas salt ingestion results in a more widespread pattern of activation, involving gustatory and viscerosensory nuclei of the $\mathrm{PB}$ complex. These findings suggest that the forebrain receives two very different types of signal from the brainstem: one representing sodium need, and the others salt detection. These signals, which are transmitted to the forebrain via separate relay sites in the dorsolateral pons, arise in functionally distinct subpopulations of neurons within the NTS.

\section{Activation of the HSD2 neurons is uniquely sodium appetite-specific}

The overall activation of neurons in the NTS and AP is much greater after $\mathrm{NaCl}$ ingestion (or direct intravenous or intraperitoneal injection of hypertonic $\mathrm{NaCl}$; see Hochstenbach et al., 1993; Geerling and Loewy, 2007) than it is during chronic sodium deprivation. In contrast, NTS neurons that express HSD2 are activated specifically in association with sodium appetite, and are then rapidly inactivated after salt intake (present data; Geerling et al., 2006a; Geerling and Loewy, 2007). The HSD2 neurons remain the only phenotypically identified group in the brain with these specific properties.

Nonetheless, c-Fos activation during sodium deprivation was also evident in some non-HSD2-expressing NTS neurons. Most of this activation is found within and just lateral to the distribution of the HSD2 neurons. As shown here, A2 noradrenergic neurons account for a portion of this activation. Neurons in the A2 group, however, are clearly not activated specifically in association with sodium appetite. In contrast to the HSD2 neurons, even more neurons in this group displayed c-Fos activation after salt ingestion.

The A2 neurons are activated in response to a wide variety of stimuli, including the direct injection of hypertonic saline (Ceccatelli et al., 1989; Hochstenbach et al., 1993; Geerling and Loewy, 2007), gastrointestinal stretch (Willing and Berthoud, 1997), food intake (Rinaman et al., 1998), cholecystokinin injection (Rinaman et al., 1993), opiate withdrawal (Stornetta et al., 1993; Delfs et al., 2000), hemorrhage (Chan and Sawchenko, 1995), hypoxia (Buller et al., 1999), and immune activation (Ericsson et al., 1994). Their activation usually occurs in tandem with a much more widespread increase in the activation of non-catecholaminergic neurons in the surrounding NTS (present results; Hochstenbach et al., 1993; Stornetta et al., 1993; Geerling and Loewy, 2007). It remains possible that separate, as-yet indistinguishable subpopulations of A2 neurons are activated under separate conditions like sodium deprivation and salt ingestion, but the weight of available evidence suggests that the A2 noradrenergic neurons provide a nonspecific signal for multiple modalities of viscerosensory activation. Thus, A2 neurons are unlikely to provide information specifically related to the need for sodium.

Nonetheless, the possibility that A2 neurons do influence sodium appetite is supported by pharmacologic evidence for the modulation of salt intake by noradrenergic signaling, and by the dense axonal projection that these neurons provide to the BSTvl, a forebrain site that receives direct input from the HSD2 neurons (Geerling and Loewy, 2006b) and from forebrain circumventricular organs implicated in sodium appetite (Sunn et al., 2003). Interestingly, large lesions within the BST decrease the stimulation of salt intake by systemic administration of yohimbine, an alpha-2 adrenergic receptor antagonist (Zardetto-Smith et al., 1994). Conversely, sodium appetite is inhibited by intracerebroventricular administration of norepinephrine (Sugawara et al., 1999).

It remains unclear whether any other neurons in the NTS provide a sodium appetite-specific signal like that of the HSD2 neurons. A small number of other c-Fos-positive nuclei are usually found intermingled with the HSD2 neurons after sodium deprivation (see Fig. 4B here, and fig. 3A of Geerling et al., 2006a). Also, both the pre-LC and PBel-i appear to receive input from many other neurons in the NTS, not just the HSD2 neurons (Geerling and Loewy, $2006 \mathrm{~b}$ ). Based on these two observations, the HSD2 neurons may represent a specialized, aldosterone-sensitive subset within a larger population of NTS neurons that are activated by sodium deficiency, transmitting a combined signal for sodium need to an overlapping set of efferent targets. Evaluating this possibility will require new celltype-specific markers to label additional functional subpopulations like the HSD2 neurons.

\section{HSD2 neurons project to the pre-LC and PBel-i, which are similarly activated during sodium deprivation}

The pre-LC is a poorly understood site that lies just medial to what is traditionally regarded as the PB complex. It receives a dense axonal projection from neurons in the NTS, including a substantial number of HSD2 neurons (Geerling and Loewy, 2006b). Along its lateral border, neurons in this site intermingle with the large, distinct neurons of the $\mathrm{MeV}$. Other than this, however, neurons in the pre-LC are not reliably distinguishable from surrounding cell populations in the dorsal pontine tegmentum based on Nissl cytoarchitecture alone, nor are we aware of a marker molecule that demarcates this site. The identification of such a marker would greatly benefit future studies of the pre-LC. Its neurons are closely surrounded by a number of other neuronal populations, including another group just medial to $\mathrm{MeV}$ and referred to as the "precoeruleus," which has been implicated in REM sleep regulation ( $\mathrm{Lu}$ et al., 2006), as well as a medially adjacent (and possibly overlapping) group of neurons that strongly express neuropeptide S (Xu et al., 2004), and a population of GABAergic interneurons that innervate the caudally adjacent LC (Aston-Jones et al., 2004). Due to the paucity of cell-type-specific immunohistochemical markers for clearly distinguishing these and other intermixed populations inside this densely packed region, we cannot rule out the possibility that some or all of the neurons in the functional cluster we have identified as "pre-LC" are also members of one of these other groups.

At present, the pre-LC can be distinguished from surrounding neuronal populations by 1) its dense field of axon terminals originating from the NTS (Geerling and Loewy, $2006 \mathrm{~b}$ ) and 2) its conspicuous cluster of c-Fos-labeled nuclei that appear after prolonged sodium deprivation, as shown here. The unambiguous correspondence within the pre-LC between the axonal varicosities arising from the 
A

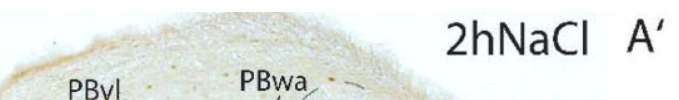

\section{$A^{\prime}$}
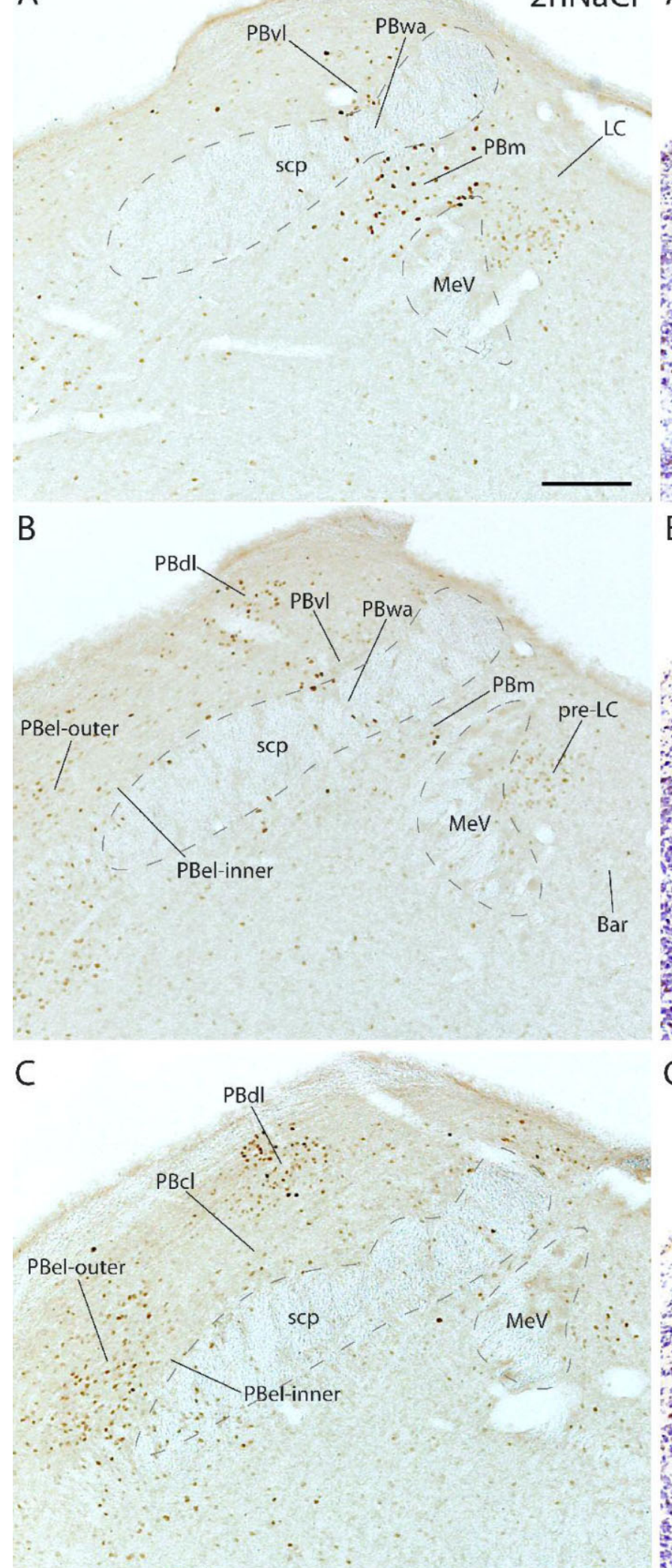

$\mathrm{B}^{\prime}$

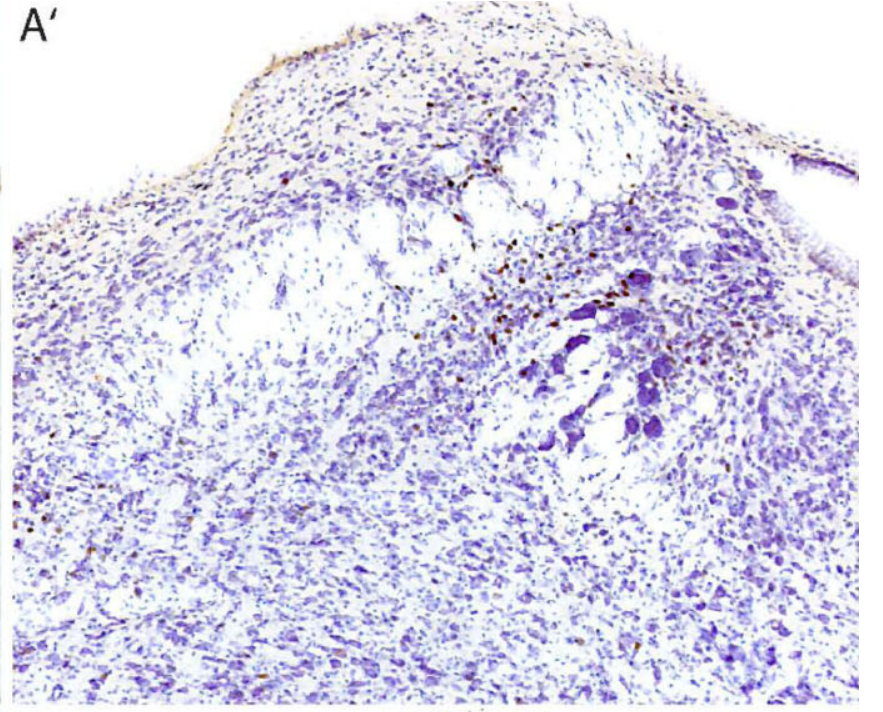
$\because \cdots+1$
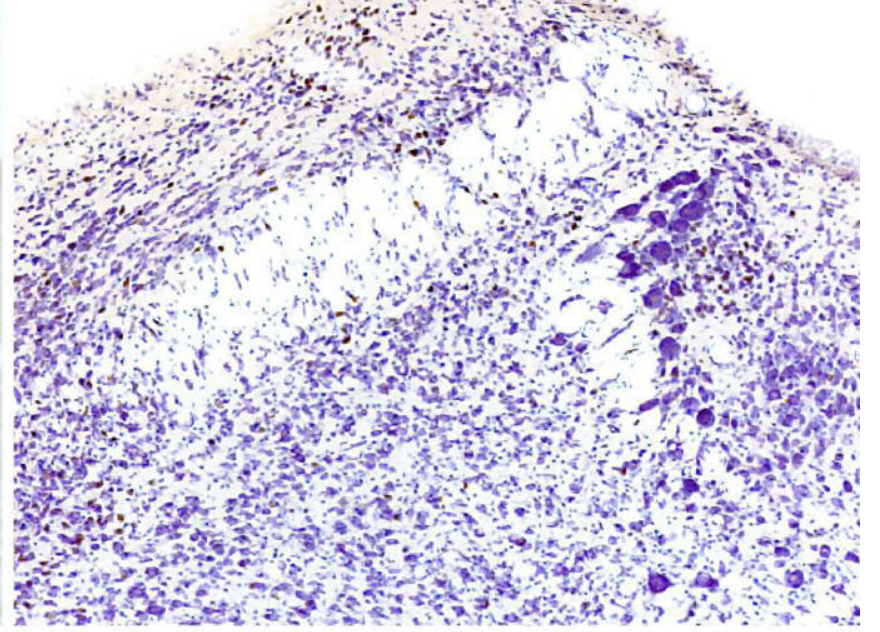

$C^{\prime}$
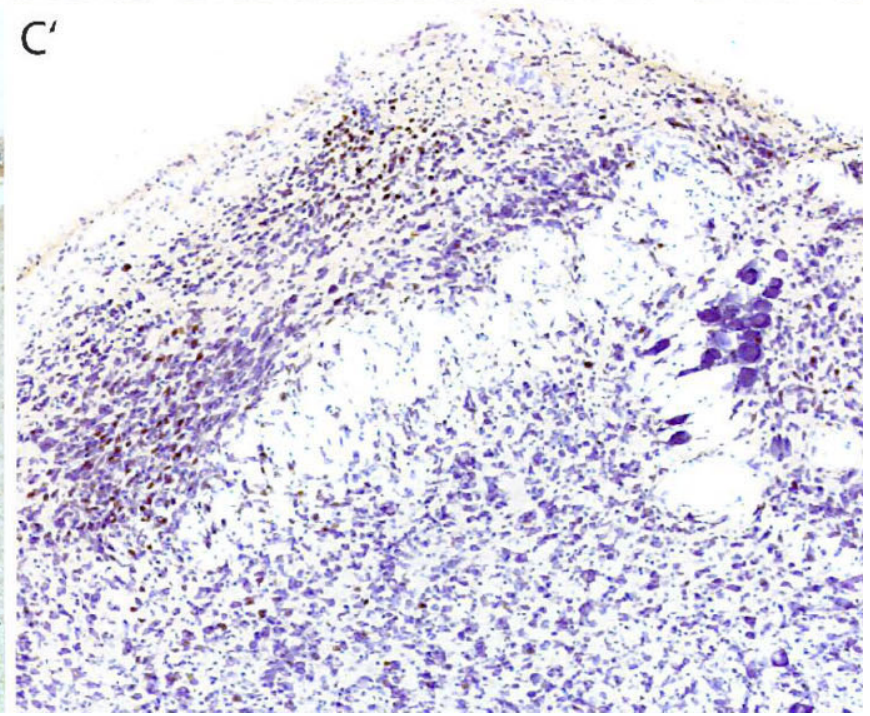

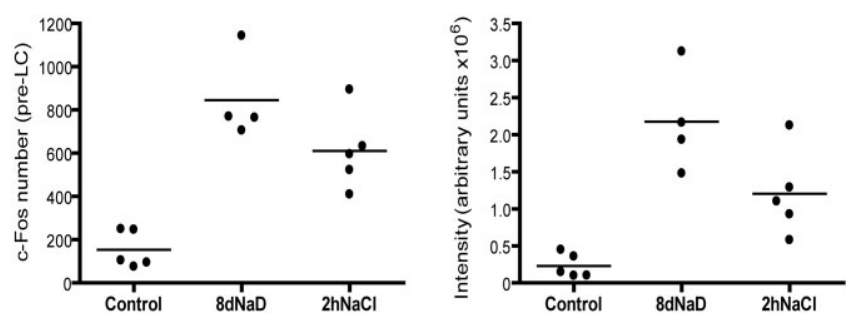

Fig. 18. Neurons in the pre-LC, similar to HSD2 neurons in the NTS, showed a marked increase in nuclear c-Fos labeling after 8-day sodium deprivation $(P<0.001$ vs. controls, compared as either the number or intensity of c-Fos labeling). After salt intake a decrease in the overall intensity of c-Fos labeling within this ROI (but not the decrease in its total number of labeled nuclei) was statistically significant $(P<0.05$; note: all other significant differences in immunoreactive intensity in this study were accompanied by a similarly significant difference in total number). Both the number and intensity of c-Fos-labeled nuclei in after $2 \mathrm{~h} \mathrm{NaCl}$ were still significantly elevated above control levels $(P<0.05, P<0.01$, respectively).

HSD2 subregion of the NTS and the cluster of c-Fos labeling here after sodium deprivation suggests that neurons in the pre-LC are directly stimulated by HSD2 neurons. This possibility is also supported by the reduced c-Fos labeling in both the HSD2 group and in the pre-LC after rats consumed saline.

The exact downstream consequences of neuronal activation in the pre-LC remain uncertain, and only preliminary data exist regarding its output connections to sites in the forebrain (Luppi et al., 1995; Geerling and Loewy, 2006b). Nonetheless, the sodium deprivation-induced activation of this site is consistent with the possibility that it plays an important role in stimulating some behavioral aspect of sodium appetite. Consistent with this hypothesis, a virtual absence of saline ingestion was observed in sodium-deficient rats after lesions that targeted the medial $\mathrm{PB}$, which appeared to involve part or all of the pre-LC (Flynn et al., 1991; Scalera et al., 1995). Due to the difficulty in restricting such lesions to individual functional groups of neurons within this region of the $\mathrm{PB}$ complex, the question of which specific subnuclei are func-

Fig. 17. A-C: c-Fos immunoreactivity at three rostrocaudal levels of the dorsolateral pons in a representative case from the group that was given $2 \mathrm{~h}$ access to concentrated saline $(2 \mathrm{hNaCl})$ after 8 -day of sodium deprivation (\#8069). This rat drank a total of $9.5 \mathrm{~mL}$ of $3 \%$ $\mathrm{NaCl}$ during this $2 \mathrm{~h}$ period $(8.5 \mathrm{~mL}$ within the first 30 minutes). $\mathrm{A}^{\prime}-\mathrm{C}^{\prime}$ : The same three sections counterstained with thionin to reveal surrounding cytoarchitecture. These three incremental rostrocaudal levels of the PB complex roughly correspond to those shown for control and $8 \mathrm{dNaD}$ groups in Figures 15 and 16. First, the gustatory nuclei at caudal levels of the PB complex contained a prominent collection of intense c-Fos labeling, shown primarily in A, and continuing rostrally into B. Second, a number of c-Fos-labeled nuclei remained in the pre-LC, although their immunoreactive intensity was reduced (shown in $\mathrm{B}$, but also extending caudally into $\mathrm{A}$, due to a difference in section angle relative to the examples shown in Fig. 16). Third, extensive c-Fos labeling was scattered throughout all mid-rostral levels of the lateral PB (LPBN), including somewhat more prominent collections in the outer subdivision of $\mathrm{PBel}$ and in $\mathrm{PBdl}$, which receive dense axonal projections from neurons in the regions of the medial NTS and AP that exhibited intense c-Fos labeling in the same cases (see Fig. 6). Scale bar $=200 \mu \mathrm{m}$ in A (applies to all).

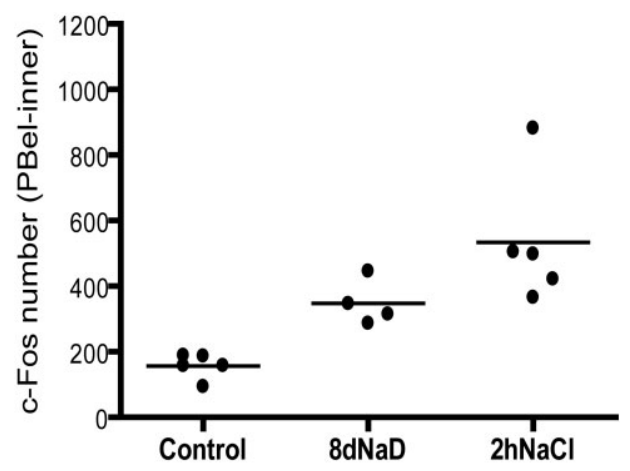

Fig. 19. Sodium-deprived rats showed a statistically significant increase in the number of c-Fos-labeled nuclei within the ROI encompassing the PBel-i $(P<0.05$ vs. controls). In the $2 \mathrm{~h}$ saline-access group, both the number and intensity of c-Fos-labeled nuclei remained elevated over control levels $(P<0.01, P<0.05$, respectively), although neither changed significantly versus sodium-deprived rats.

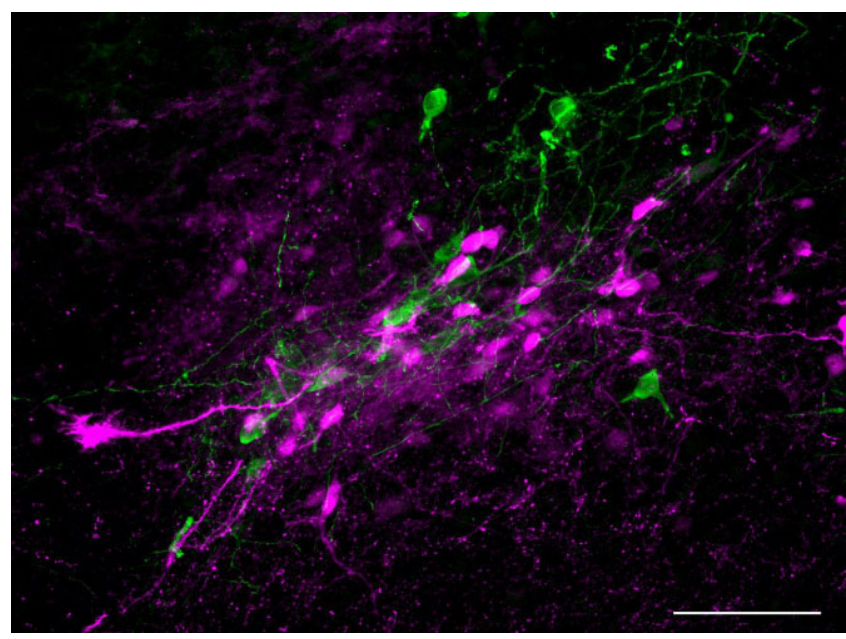

Fig. 20. PHAL (magenta) was injected directly into the distribution of NTS neurons immunoreactive for HSD2 (green) in this 8-day sodium-deprived case (\#8616). This PHAL injection site was centered ventrolateral to the rostral extent of the AP, within the distribution of the dense rostral group of HSD2 neurons. Scale bar $=100 \mu \mathrm{m}$.

tionally involved in stimulating sodium appetite remains unanswered.

Although much remains to be learned about the connections and functions of the pre-LC, somewhat more information is available regarding the inner subdivision of PBel. This site receives direct input from the HSD2 neurons and from other cells in their subregion of the NTS (Herbert et al., 1990; Geerling and Loewy, 2006b), including neurons that express galanin or corticotropinreleasing hormone (Herbert and Saper, 1990). Neurons in the PBel-i project, in turn, to the lateral and lateral capsular subdivisions of the central nucleus of the amygdala (CeA; Bernard et al., 1993; Geerling and Loewy, 2006a), as well as to the BSTvl (Alden et al., 1994; Geerling and Loewy, 2006b).

After 8-day dietary sodium deprivation, increased c-Fos labeling was found only within an extremely restricted subregion of PBel-i. The medial clustering of these acti- 

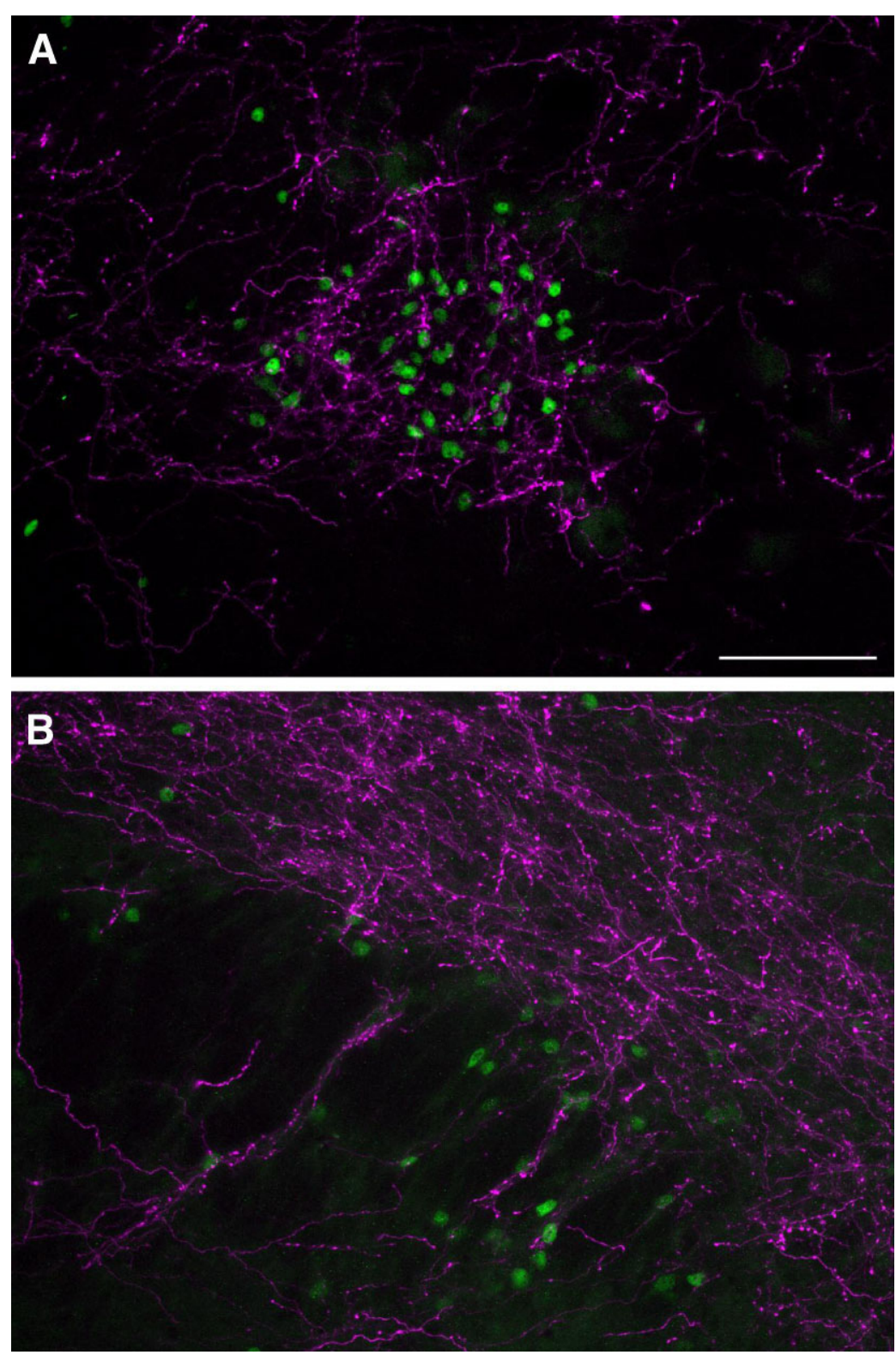

Fig. 21. A: Sodium deprivation-activated neurons in the pre-LC (nuclear c-Fos labeling, shown in green) are entangled in a dense meshwork of axons and boutons from the NTS, ipsilateral to an injection of the anterograde neural tracer PHAL (magenta; case 8616 , see injection site in Fig. 20). For anatomic orientation, faint green autofluorescence can also be seen in the large neurons of the adjoining mesencephalic nucleus of the trigeminal nerve $(\mathrm{MeV})$, arching around the lateral border of the c-Fos labeled nuclei in the pre-LC. B: Sodium deprivation-activated neurons in the PBel-i (nuclear c-Fos, in green)

vated neurons-adjacent to and penetrating into the superior cerebellar peduncle-suggests that the subregion of this nucleus influenced by HSD2 neurons is even smaller than we predicted based on anterograde and retrograde tracing data from this region (Geerling and Loewy, 2006b).

The inner and outer subdivisions of PBel show distinct patterns of expression for a number of neuropeptides and peptide receptors (Hermanson and Blomqvist, 1997; Hall- are also surrounded by PHAL-labeled axons and terminals from the NTS (magenta); case 8616, injection site shown in Fig. 20. Note the cascades of c-Fos-labeled nuclei and attendant PHAL-labeled axons penetrating into the lateral border of the scp, similar to the distribution of sodium deprivation-induced c-Fos activation shown above (Fig. $16)$ and similar to our prior depiction of axons from the HSD2 subregion of the NTS (Geerling and Loewy, 2006b). Scale bar $=100 \mu \mathrm{m}$ in A (applies to B).

beck et al., 1999; Engstrom et al., 2001; Engblom et al., 2004; Paues et al., 2006). These two subdivisions also receive axonal projections from different populations of neurons in the NTS and AP (Herbert et al., 1990; Herbert and Saper, 1990). The outer subdivision exhibits robust activation in response to a variety of postingestive negative feedback signals (similar to the c-Fos activation shown here in rats that ingested large volumes of hypertonic saline) and also signals of overt visceral malaise, 


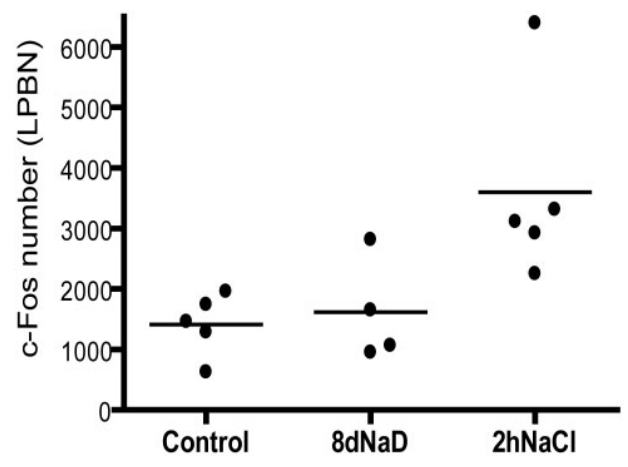

Fig. 22. When measured across the overall LPBN, a significant increase in c-Fos activation was found only after salt intake $(P=0.02$ vs. controls).

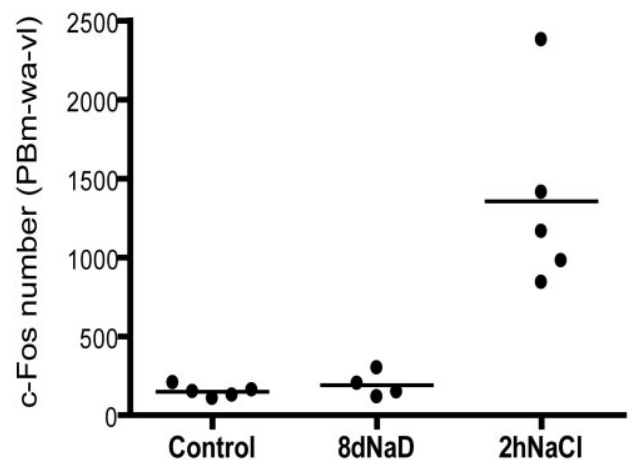

Fig. 23. Rats that drank concentrated $\mathrm{NaCl}$ exhibited intense c-Fos labeling in gustatory-recipient nuclei within the caudal PB (a band of neurons extending through PBm, PBwa, and PBvl). This activation represented a significant increase over controls and sodium-deprived rats $(P<0.001$ and $P<0.01$, respectively).

including systemic administration of lithium or lipopolysaccharide (Paues et al., 2006). This activation is mediated by vagal and humoral signals that stimulate large numbers of visceroceptive and chemoreceptive neurons in the NTS and AP, which exhibit c-Fos activation under the same conditions (Yamamoto et al., 1992; Swank and Bernstein, 1994; Sagar et al., 1995) and project heavily to the outer subdivision of PBel (Shapiro and Miselis, 1985; Herbert et al., 1990). In contrast to this well-known viscerosensory relay site, the inner subdivision of PBel has been said to have no known physiological stimulus (see Discussion, Paues et al., 2006). The present results establish that at least one condition-prolonged sodium deficiencystimulates neurons in its medial extent, likely as a result of the activation of HSD2 neurons in the NTS.

\section{Salt intake potently activates neurons in the NTS, AP, and PB complex}

By far the greatest activation in the NTS, AP, and PB in the present experiments was found after salt intake. The patterns of pronounced c-Fos labeling after the ingestion of concentrated $\mathrm{NaCl}$ indicate that these brainstem regions transmit distinct feedback signals representing the initial taste of salt and for the subsequent detection of ingested sodium.

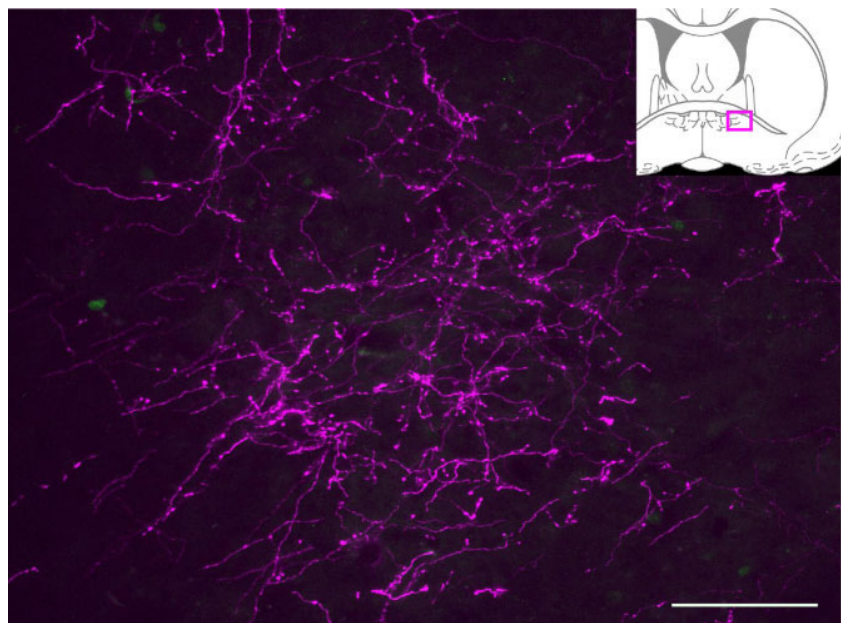

Fig. 24. In contrast to the HSD2 neuron-innervated sites in the pons shown above, the ventrolateral bed nucleus of the stria terminalis (BSTvl) contained virtually no c-Fos labeling in 8-day sodiumdeprived rats. This photomicrograph shows immunoreactivity for c-Fos (green) along with PHAL anterograde axonal labeling from the NTS (magenta), which primarily overlaps the fusiform subnucleus within BSTvl (Ju and Swanson, 1989; Geerling and Loewy, 2006b). This photomicrograph was taken from the same PHAL-injected, 8-day sodium-deprived case (\#8616, injection site shown in Fig. 20) from which photomicrographs of heavy c-Fos labeling and axonal labeling are shown in the pre-LC and PBel-i (Fig. 21). Similar to this example, no animal in this study contained more than a few scattered, faintly labeled c-Fos nuclei in this region. Scale bar $=100 \mu \mathrm{m}$.

Besides providing postingestive negative feedback information to rostral brain sites (discussed below), the pronounced activation of neurons in the AP and medial NTS may also serve to inactivate the HSD2 neurons after salt intake. In the present study, HSD2 neuronal activation during sodium deprivation was reduced shortly after the ingestion of concentrated saline, as we have found previously (Geerling et al., 2006a; Geerling and Loewy, 2006c). After just 1 hour, c-Fos immunoreactivity disappeared in the majority of these neurons. A similarly rapid decrease in c-Fos-immunoreactivity has been observed in a group of dehydration-activated neurons in the hypothalamus following an analogous sequence of water deprivation and brief access to water (Wotus et al., 2007). The inactivation of HSD2 neurons after salt ingestion may be mediated by salt-activated neurons in the surrounding NTS and AP, either directly or via inhibitory interneurons (see Sequeira et al., 2006).

Our results agree with previous findings regarding the activation of brainstem sites by gustatory and viscerosensory postingestive signals. For example, Yamamoto et al. (1993) observed moderate increases in c-Fos expression in $\mathrm{PBm}, \mathrm{PBel}$, and $\mathrm{PBcl}$ after rats ingested smaller volumes of less-concentrated saline, in the absence of sodium appetite. The c-Fos activation in $\mathrm{PBm}$, but not in $\mathrm{PBel}$ or $\mathrm{PBcl}$, could be prevented by blocking intraoral sodium channels with amiloride, indicating that activation of this nucleus was related to taste stimulation, whereas the activation of PBel and $\mathrm{PBcl}$ was driven by nongustatory postingestive signals delivered by visceral afferents from the gut (Yamamoto et al., 1993). 
That intraoral $\mathrm{NaCl}$ activates neurons in the caudal PBm was later confirmed by Travers (2002), who also showed that, surprisingly, NaCl taste does not increase c-Fos labeling within gustatory-recipient neurons in the rostral NTS (rNTS). In those experiments, hypertonic saline $(0.3 \mathrm{M}, \approx 2 \% \mathrm{NaCl})$ did not alter c-Fos labeling in the rNTS relative to control stimulation with water, despite the consistent increases stimulated by bitter, sour, and sweet tastants (Travers, 2002). We did not quantify c-Fos labeling in the rNTS in the present experiments, but we did observe that, similarly, the low level of c-Fos labeling here remained qualitatively unchanged, even after the ingestion of hypertonic $\mathrm{NaCl}$ ( $\mathrm{NaCl}$-access cases did, however, exhibit greatly increased c-Fos activation in the adjacent oral premotor region of the dorsal medullary reticular formation; data not shown). It is unclear why the well-characterized salt-taste-activated gustatory neurons in rNTS, which transmit salt-taste information from peripheral gustatory afferents to $\mathrm{PBm}$, do not exhibit a detectable change in c-Fos expression after ingestion of hypertonic saline, but this discrepancy reinforces the point that only limited inferences can be made when no increase in c-Fos expression is found (Dragunow and Faull, 1989; Hoffman et al., 1993).

Regarding postingestive signaling, the present experiments did not specifically address the viscerosensory mechanisms responsible for the substantial activation of neurons in the caudal NTS and AP. We and others have shown that intraperitoneal or intravenous injection of hypertonic saline causes widespread neuronal c-Fos expression in a pattern similar to that shown here (including the activation of a subset of A2 noradrenergic neurons; Ceccatelli et al., 1989; Hochstenbach et al., 1993; Geerling and Loewy, 2007). We also observed a similar pattern of c-Fos activation in animals that ingested $3 \% \mathrm{NaCl}$ following chronic mineralocorticoid treatment (Geerling and Loewy, 2006c) or hypovolemia (unpubl. obs. J.C.G., A.D.L.).

Some of this activity could be driven by the activation of cerebral osmoreceptors and/or sodium-sensors in the forebrain (stimulated by a rise in the plasma concentration of sodium shortly after the ingestion of $3 \% \mathrm{NaCl}$ ), which may influence neurons in the NTS through the descending projections of their target neurons in the paraventricular nucleus of the hypothalamus (van der Kooy et al., 1984). The majority of synaptic input to this part of the NTS, however, is provided by the vagus nerve. The strong correlation we found between the volume of saline ingested and the degree of c-Fos activation in the NTS and AP (along with the similar patterns of c-Fos activation found after ingestion or intraperitoneal injection of hypertonic saline) are consistent with the activation of vagal afferents that detect distention and hypertonicity within the gut and/or portal vasculature. At this point, the relative importance of ingested fluid volume (sensed as gastrointestinal distention by mechanosensitive vagal afferents) versus solute concentration (sensed via osmosensitive vagal afferents in the gut and/or portal vasculature) versus detection of the sodium ion itself (Morita et al., 1997; Stricker et al., 2007) remains unclear. The subsequent elevation in plasma sodium concentration may also stimulate neurons in the AP directly.

Rats without an AP will ingest enormous volumes of concentrated saline, with or without sodium deprivation (Contreras and Stetson, 1981; Watson, 1985; Edwards et al., 1993; Wang and Edwards, 1997; Curtis et al., 1999). AP neurons project directly to the underlying NTS and to a restricted stripe through the LPBN, targeting the outer subdivision of $\mathrm{PBel}$ and extending into PBcl (Shapiro and Miselis, 1985). Large lesions of the LPBN significantly increase the volume of water ingested by thirsty rats (Ohman and Johnson, 1986; Edwards and Johnson, 1991), but apparently do not increase the saline intake of sodium-deficient animals (Johnson and Thunhorst, 1997). Nonetheless, injecting various peptidergic and monoaminergic neurotransmitter agonists and antagonists directly into the LPBN can substantially alter the amount of concentrated saline rats will ingest in response to various stimuli for sodium appetite (Menani et al., 1996, 1998, 2002; Menani and Johnson, 1998; Fratucci De Gobbi et al., 2001; Andrade et al., 2004, 2006; Callera et al., 2005; De Gobbi et al., 2005). Collectively, these findings indicate that postingestive signals from the caudal NTS and AP ascend via relay nuclei in the LPBN and mediate a key component of the feedback regulation of salt-ingestive behavior.

The nonspecific term "LPBN" lumps together multiple anatomically distinct nuclei located dorsolateral to the scp. These nuclei, found at mid-rostral and rostral levels of the PB complex, include PBel, PBcl, and PBdl (Fulwiler and Saper, 1984). With respect to sodium deprivation and salt ingestion, the present findings suggest that this complex exhibits significant functional heterogeneity (e.g., PBel-inner vs. -outer), and provide a more precise anatomical framework for the interpretation of previous and future experimental manipulations in this region.

\section{Potential implications: input pathways that may regulate sodium appetite}

Previously, we hypothesized that separate groups of neurons in the NTS and PB, via separate ascending pathways, transmit two distinct types of information for sodium appetite: sodium need and salt detection (Geerling and Loewy, 2006b). During sodium deprivation the HSD2 neurons, via their efferent target neurons in the pre-LC and PBel-i, transmit a signal specifically associated with sodium need (Fig. 25A). During and after the ingestion of salt, gustatory pathways (relayed through the caudal medial PB) and viscerosensory pathways (relayed through the rostral lateral $\mathrm{PB}$ ) provide two qualitatively different signals for salt detection (Fig. 25B,C).

First, taste detection of salt activates gustatory nerves that innervate the rNTS (Hamilton and Norgren, 1984). Then, taste-recipient neurons in the rNTS transmit this information to the forebrain via gustatory relay nuclei in the caudal PB (Norgren and Leonard, 1971; Norgren, 1976). The taste of salt becomes a powerful stimulus after sodium-deprivation, driving stereotyped, highly motivated ingestive motor behavior that is not observed in sodium-replete animals. This motivational value of salt is completely dependent on taste detection, as transection of peripheral gustatory nerves (Frankmann et al., 1996; Roitman and Bernstein, 1999), the rostral NTS (Flynn et al., 1991), and lesions of a caudal-medial region of the PB complex (Flynn et al., 1991; Scalera et al., 1995) markedly decrease or completely eliminate this motivated ingestive behavior.

Subsequently, postingestive detection of hypertonic saline inside the gut provides an early, negative feedback signal that tells the animal to stop drinking. Postingestive 
A

\section{"Low on sodium"} (HSD2 neurons)

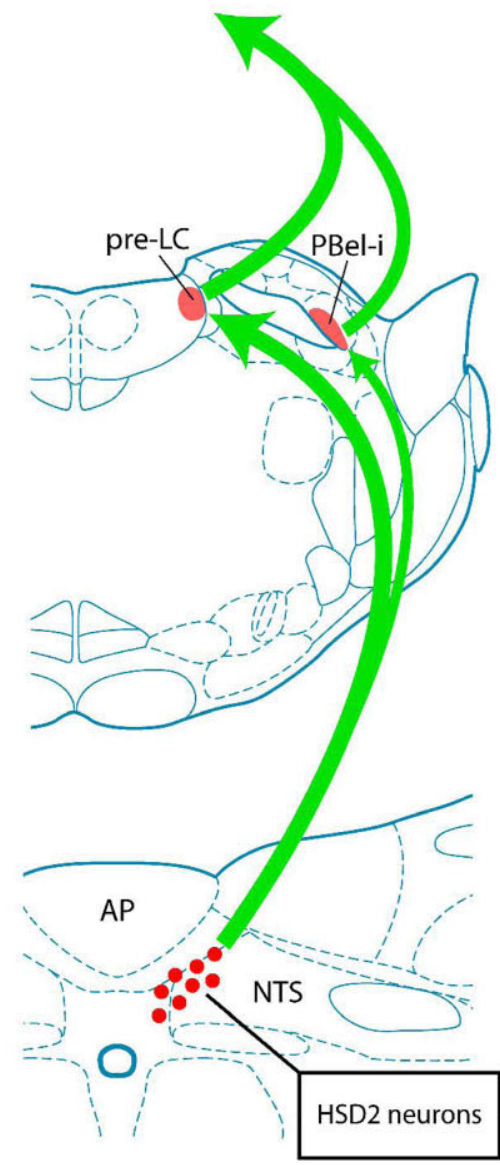

Sodium need

B

"Salt located" (taste input)

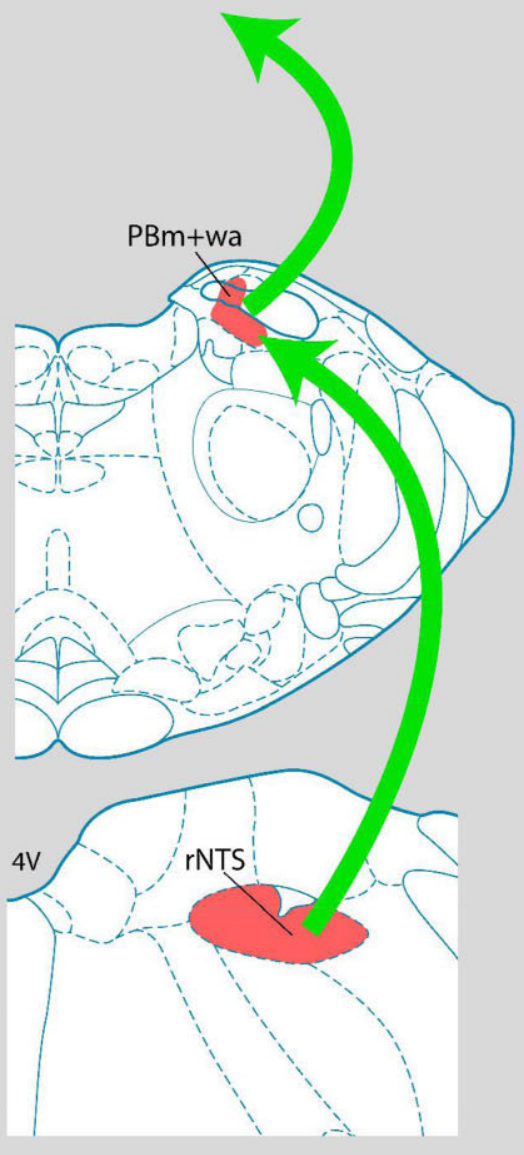

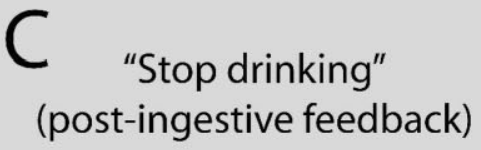

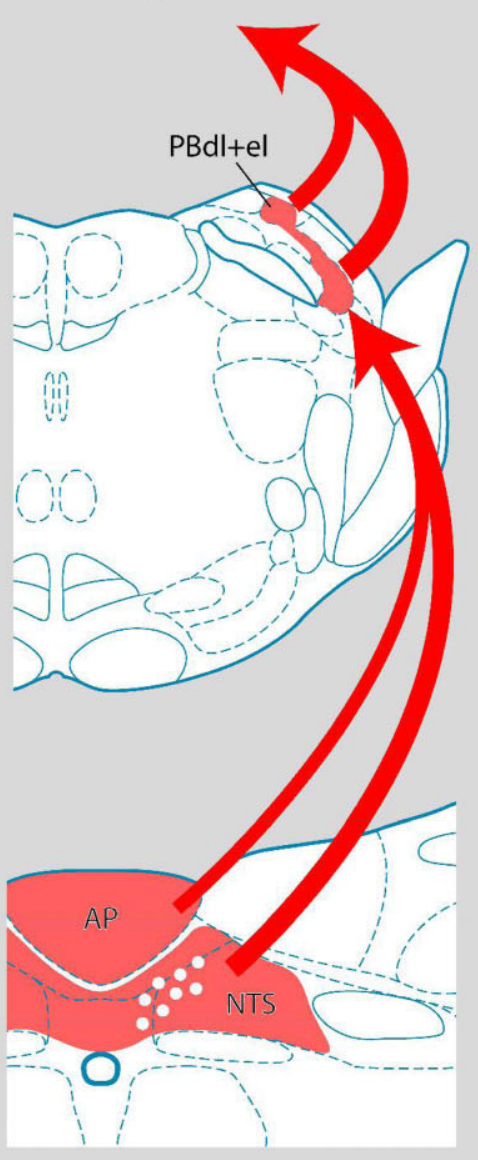

Salt detection

Fig. 25. Separate pathways from the brainstem to the forebrain transmit information specifically associated with (A) sodium deficiency, (B) gustatory detection of salt, and (C) postingestive detection of ingested salt. A: Prolonged sodium deprivation activates the HSD2 neurons, which directly activate two restricted nuclei in the pons, the pre-LC and PBel-i (shown here at the same rostrocaudal level for simplicity), both of which provide substantial axonal projections to subcortical sites in the forebrain, largely within the extended amygdala complex (Bernard et al., 1993; Alden et al., 1994; Geerling and Loewy, 2006b). These signaling pathways, which are activated specifically in association with sodium need, may transmit information important for the stimulation of salt ingestion or other behavioral changes associated with sodium appetite. Note that salt intake is also influenced by other hypovolemia-associated signals that are not discussed here, such as the activation of forebrain circumventricular organs by angiotensin II (Fitts et al., 2004). B: Taste detection of salt is transmitted to the rostralmost part of the NTS (rNTS) by gustatory nerves from the oropharynx (Hamilton and Norgren, 1984). Tasteactivated neurons in the rNTS project to a caudal region of the PB surrounding the medial scp, the "pontine taste area" of Norgren (Norgren and Leonard, 1971; Norgren and Pfaffmann, 1975; Herbert et al., 1990). In turn, the axons of gustatory-recipient neurons in the $\mathrm{PB}$ project to limbic and thalamic sites in the forebrain (Travers et al.,
1987; Bernard et al., 1993; Alden et al., 1994; Bester et al., 1999; Geerling and Loewy, 2006b). During prolonged sodium deficiency, these gustatory salt-detection signals become a more powerful motivator for ingestive motor behavior (Roitman and Bernstein, 1999). C: As saline is consumed, neural and humoral postingestive signals from the gut and blood are detected by neurons in the caudal NTS and AP. First, viscerosensory afferent fibers in the vagus nerve are stimulated by gut distention (proportional to the volume of ingested fluid), by luminal hyperosmolarity (due to the hypertonicity of $3 \% \mathrm{NaCl}$ ), and probably also by sodium-specific sensors within the portal vasculature as sodium is absorbed (Morita et al., 1997). These activated vagal afferents stimulate neurons in the NTS as well as cells in the AP, some of which can directly monitor humoral variables such as plasma osmolarity. Many of these neurons in the caudal medial NTS and AP project to nuclei within the LPBN, including primarily the PBel, PBcl, and PBdl (Herbert et al., 1990). In turn, neurons in these LPBN nuclei transmit this information to a variety of limbic and hypothalamic targets in the forebrain (Bernard et al., 1993; Bester et al., 1997), providing negative feedback signals that inhibit ingestive behavior (Contreras and Stetson, 1981; Menani et al., 1996, 1998, 2002; Johnson and Thunhorst, 1997; Menani and Johnson, 1998; Fratucci De Gobbi et al., 2001; Andrade et al., 2004, 2006). Brain drawings are modified from Paxinos and Watson (2005). 
information is transmitted directly to the NTS and AP from the stomach, proximal duodenum, and other subdiaphragmatic structures by the vagus nerve (Norgren and Smith, 1988). Next, neurons in the NTS and AP transmit this viscerosensory information to the forebrain, primarily via relay nuclei in the LPBN (Herbert et al., 1990; Bernard et al., 1993; Alden et al., 1994; Bester et al., 1997). If these postingestive negative-feedback signals are attenuated (by destroying specific vagal afferent fiber subtypes) or eliminated (by draining ingested fluid from the stomach via a gastric fistula), salt-hungry rats will continue to drink large volumes of saline for an extended period of time (Frankmann et al., 1996; Curtis and Stricker, 1997; Roitman et al., 1997).

As outlined above, signals from the medulla associated with either sodium need or salt detection are relayed to the forebrain through largely separate relay sites in the PB complex, but minor overlap exists in this region between gustatory projections from the rostral NTS and viscerosensory projections from the caudal NTS (Hermann and Rogers, 1985; Karimnamazi et al., 2002). Thus, some early integration of salt-regulatory signals could occur directly within the PB. In this regard, it is interesting that after c-Fos activation increased in the PBel-i during sodium deprivation, in contrast to the pre-LC, it was sustained with a tendency toward even greater activation after salt ingestion. It is unclear, however, whether the same subpopulation of PBel-i neurons were activated under each condition; for example, intermixed subpopulations of neurons within PBel-i could exhibit separate activity patterns that are indistinguishable based on their spatial location alone (similar to the HSD2 neurons and other intermingled groups in the medial NTS). Nonetheless, if this site received input only from sodium deficiency-activated neurons in the NTS like the HSD2 group, its c-Fos immunoreactivity would have decreased after salt intake, so these findings suggest that PBel-i may contain neurons that integrate signals for sodium need with those for salt detection. In pre-LC, on the other hand, the significant decrease in c-Fos intensity after salt intake (and the tendency toward a decrease in the number of c-Fos-labeled nuclei) suggests that this larger HSD2-neuron-innervated nucleus is activated primarily in association with sodium need.

\section{Forebrain integration of sodium appetite- associated input signals}

Specific nuclei within the central extended amygdala complex-particularly the BSTvl and CeA-may be key sites for integrating the appetitive signals associated with sodium need and salt detection. This hypothesis is based, in part, on their input from sodium appetite-associated groups including the HSD2 neurons, pre-LC, and PBel-i (Reilly et al., 1994; Johnson et al., 1999; Sakai et al., 2000; Sunn et al., 2003; Geerling and Loewy, 2006a,b). For example, in addition to direct input from the HSD2 neurons and the A2 noradrenergic neurons in the NTS, the BSTvl also receives input from 1) the same two $\mathrm{PB}$ subnuclei targeted by the HSD2 neurons (Geerling and Loewy, $2006 \mathrm{~b})$; 2) neurons in the caudal-medial (gustatory) region of the PB (Alden et al., 1994; Geerling and Loewy, 2006b); 3) forebrain circumventricular organs that detect bloodborne sodium appetite stimuli such as angiotensin II (Sunn et al., 2003); and 4) appetite-related peptidergic neurons in the arcuate nucleus of the hypothalamus (Bag- nol et al., 1999). Also supporting a role for these forebrain nuclei in the regulation of sodium appetite, large lesions in the BST or the CeA significantly attenuate sodium appetite (Reilly et al., 1994; Zardetto-Smith et al., 1994).

For these reasons, the consistent lack of BSTvl c-Fos activation in sodium-deprived cases was quite unexpected. Although this finding is consistent with a lack of activation in this site, the absence of c-Fos, as noted above, cannot be interpreted as evidence for inactivity because its sensitivity as a marker for neuronal activity varies dramatically with neuronal phenotype and stimulus intensity (Dragunow and Faull, 1989; Hoffman et al., 1993). It is difficult to explain why activation of the HSD2 neurons is associated with c-Fos labeling in their efferent target sites in the pons (pre-LC and PBel-i), but not in the basal forebrain (BSTvl), but a few differences between these regions may be worth noting.

First, the BSTvl, a component of the striatal-derived extended amygdala complex, is composed of a different cellular phenotype-GABAergic neurons, some of which coexpress neuropeptides like corticotropin-releasing hormone (Dong et al., 2001). Second, the BSTvl receives input from a wide variety of sites in the forebrain and brainstem, allowing that, in addition to stimulation by the HSD2 neurons, it may be subject to simultaneous inhibitory input from sites that do not inhibit the pre-LC or PBel-i. Third, the BSTvl, unlike these two pontine sites, receives a heavy input projection from many A2 noradrenergic neurons in the NTS. Besides the HSD2 and A2 neurons, only a small number of other, unlabeled neurons in the NTS project to this site (Geerling and Loewy, 2006b). In contrast, the PB complex receives input from relatively few A2 noradrenergic neurons (Milner et al., 1986; Gaykema et al., 2007; unpubl. obs. J.C.G., A.D.L.), despite massive input projections from a considerably larger population of neurons in the NTS (Herbert and Saper, 1990). If A2 neurons that project to the BSTvl are among those that are activated by sodium deprivation, they may exert an inhibitory influence, decreasing the excitatory effect of HSD2 neurons in this site. In forebrain slice preparations, for example, the predominant effect measured in response to norepinephrine in the BSTvl is $\alpha 2$-adrenoreceptor-mediated inhibition of axonal glutamate release (Egli et al., 2005).

It will be important to determine 1) whether the lack of c-Fos in the BSTvl after sodium deprivation reflects a true lack of neuronal activation, and, if so, 2) what are the reasons for and implications of this inactivity. Answers to these two questions would provide important functional information about the BSTvl, which is positioned to produce integrated changes in arousal, ingestive behavior, neuroendocrine function, and autonomic activity based on its unique pattern of output connections (see Dong et al., 2001; Yoshida et al., 2005).

Another site that receives input from HSD2 neurons, the pre-LC, and PBel-i is the CeA (Geerling and Loewy, 2006a). This site is implicated in the regulation of sodium appetite (reviewed in Johnson et al., 1999; see also discussion in Geerling and Loewy, 2006a). Like the BSTvl, however, this region did not exhibit any change in c-Fos labeling after sodium deprivation, although c-Fos labeling in the CeA did increase consistently after salt intake (unpubl. obs.). Detailed analysis of c-Fos labeling throughout the forebrain-both during sodium deprivation and after salt intake-will be an important topic for future investi- 
gation. Unfortunately, these preliminary findings suggest that c-Fos labeling will provide only limited information regarding downstream activity in the forebrain limbic sites that ultimately receive and integrate information from the sensory pathways activated by sodium deprivation.

\section{CONCLUSIONS}

Separate groups of neurons within the NTS and AP transmit distinct sodium-related signals to the forebrain via separate relay nuclei in the $\mathrm{PB}$. As illustrated in Figure 25, these distinct patterns of axonal input and c-Fos activation in the medulla and pons suggest that the brainstem provides both stimulatory and inhibitory signals for salt intake via parallel, functionally opposed pathways.

\section{ACKNOWLEDGMENTS}

We thank Xay Van Nguyen for expert surgical and histological assistance.

\section{LITERATURE CITED}

Alden M, Besson JM, Bernard JF. 1994. Organization of the efferent projections from the pontine parabrachial area to the bed nucleus of the stria terminalis and neighboring regions: a PHA-L study in the rat. J Comp Neurol 341:289-314.

Andrade CA, Barbosa SP, De Luca LA Jr, Menani JV. 2004. Activation of alpha2-adrenergic receptors into the lateral parabrachial nucleus enhances $\mathrm{NaCl}$ intake in rats. Neuroscience 129:25-34.

Andrade CA, De Luca LA Jr, Colombari DS, Menani JV. 2006. Alpha(2)adrenergic activation in the lateral parabrachial nucleus induces $\mathrm{NaCl}$ intake under conditions of systemic hyperosmolarity. Neuroscience 142:21-28.

Aston-Jones G, Zhu Y, Card JP. 2004. Numerous GABAergic afferents to locus ceruleus in the pericerulear dendritic zone: possible interneuronal pool. J Neurosci 24:2313-2321.

Bagnol D, Lu XY, Kaelin CB, Day HE, Ollmann M, Gantz I, Akil H, Barsh GS, Watson SJ. 1999. Anatomy of an endogenous antagonist: relationship between Agouti-related protein and proopiomelanocortin in brain. J Neurosci 19:RC26

Bealer SL, Johnson AK. 1979. Sodium consumption following lesions surrounding the anteroventral third ventricle. Brain Res Bull 4:287-290.

Bernard JF, Alden M, Besson JM. 1993. The organization of the efferen projections from the pontine parabrachial area to the amygdaloid complex: a Phaseolus vulgaris leucoagglutinin (PHA-L) study in the rat J Comp Neurol 329:201-229.

Bester H, Besson JM, Bernard JF. 1997. Organization of efferent projections from the parabrachial area to the hypothalamus: a Phaseolus vulgaris-leucoagglutinin study in the rat. J Comp Neurol 383:245-281.

Bester H, Bourgeais L, Villanueva L, Besson JM, Bernard JF. 1999. Differential projections to the intralaminar and gustatory thalamus from the parabrachial area: a PHA-L study in the rat. J Comp Neurol 405:421-449.

Braun-Menendez E. 1950. Action of desoxycorticosterone on water exchange in the rat. Am J Physiol 163:701.

Buller KM, Smith DW, Day TA. 1999. NTS catecholamine cell recruitment by hemorrhage and hypoxia. Neuroreport 10:3853-3856.

Callera JC, Oliveira LB, Barbosa SP, Colombari DS, De Luca LA Jr, Menani JV. 2005. GABA(A) receptor activation in the lateral parabrachial nucleus induces water and hypertonic $\mathrm{NaCl}$ intake. Neuroscience 134:725-735.

Ceccatelli S, Villar MJ, Goldstein M, Hokfelt T. 1989. Expression of c-Fos immunoreactivity in transmitter-characterized neurons after stress. Proc Natl Acad Sci U S A 86:9569-9573.

Chae HE, Heideman PD. 1998. Water-deprived white-footed mice express c-fos on a day/night cycle graded according to the duration of deprivation. Brain Res 791:1-10.

Chan RK, Sawchenko PE. 1995. Hemodynamic regulation of tyrosine hy- droxylase messenger RNA in medullary catecholamine neurons: a c-fos-guided hybridization histochemical study. Neuroscience 66:377390

Contreras RJ, Hatton GI. 1975. Gustatory adaptation as an explanation for dietary-induced sodium appetite. Physiol Behav 15:569-576.

Contreras RJ, Stetson PW. 1981. Changes in salt intake lesions of the area postrema and the nucleus of the solitary tract in rats. Brain Res 211:355-366

Curtis KS, Stricker EM. 1997. Enhanced fluid intake by rats after capsaicin treatment. Am J Physiol 272:R704-709.

Curtis KS, Huang W, Sved AF, Verbalis JG, Stricker EM. 1999. Impaired osmoregulatory responses in rats with area postrema lesions. Am J Physiol 277:R209-219.

Daniels D, Fluharty SJ. 2004. Salt appetite: a neurohormonal viewpoint. Physiol Behav 81:319-337.

De Gobbi JI, Barbosa SP, De Luca LA Jr, Thunhorst RL, Johnson AK, Menani JV. 2005. Activation of serotonergic 5-HT(1A) receptors in the lateral parabrachial nucleus increases $\mathrm{NaCl}$ intake. Brain Res 1066 $1-9$.

De Luca LA Jr, Galaverna O, Schulkin J, Yao SZ, Epstein AN. 1992. The anteroventral wall of the third ventricle and the angiotensinergic component of need-induced sodium intake in the rat. Brain Res Bull 28 : 73-87.

Delfs JM, Zhu Y, Druhan JP, Aston-Jones G. 2000. Noradrenaline in the ventral forebrain is critical for opiate withdrawal-induced aversion. Nature 403:430-434.

Denton D. 1982. The effect of electrical stimulation and lesions of the central nervous system on salt appetite. In: The hunger for salt. Berlin: Springer. p 310-337.

Denton DA, McKinley MJ, Weisinger RS. 1996. Hypothalamic integration of body fluid regulation. Proc Natl Acad Sci U S A 93:7397-7404.

Dong HW, Petrovich GD, Watts AG, Swanson LW. 2001. Basic organization of projections from the oval and fusiform nuclei of the bed nuclei of the stria terminalis in adult rat brain. J Comp Neurol 436:430-455.

Dragunow M, Faull R. 1989. The use of c-fos as a metabolic marker in neuronal pathway tracing. J Neurosci Methods 29:261-265.

Edwards GL, Johnson AK. 1991. Enhanced drinking after excitotoxic lesions of the parabrachial nucleus in the rat. Am J Physiol 261:R10391044 .

Edwards GL, Beltz TG, Power JD, Johnson AK. 1993. Rapid-onset "needfree" sodium appetite after lesions of the dorsomedial medulla. Am J Physiol 264:R1242-1247.

Egli RE, Kash TL, Choo K, Savchenko V, Matthews RT, Blakely RD, Winder DG. 2005. Norepinephrine modulates glutamatergic transmission in the bed nucleus of the stria terminalis. Neuropsychopharmacology 30:657-668.

Engblom D, Ek M, Ericsson-Dahlstrand A, Blomqvist A. 2004. EP3 and EP4 receptor mRNA expression in peptidergic cell groups of the rat parabrachial nucleus. Neuroscience 126:989-999.

Engstrom L, Engblom D, Ortegren U, Mackerlova L, Paues J, Blomqvist A. 2001. Preproenkephalin mRNA expression in rat parabrachial neurons: relation to cells activated by systemic immune challenge. Neurosci Lett 316:165-168.

Ericsson A, Kovacs KJ, Sawchenko PE. 1994. A functional anatomical analysis of central pathways subserving the effects of interleukin-1 on stress-related neuroendocrine neurons. J Neurosci 14:897-913.

Fitts DA, Freece JA, Van Bebber JE, Zierath DK, Bassett JE. 2004. Effects of forebrain circumventricular organ ablation on drinking or salt appetite after sodium depletion or hypernatremia. Am J Physiol Regul Integr Comp Physiol 287:R1325-1334.

Flynn FW, Grill HJ, Schulkin J, Norgren R. 1991. Central gustatory lesions. II. Effects on sodium appetite, taste aversion learning, and feeding behaviors. Behav Neurosci 105:944-954.

Frankmann SP, Sollars SI, Bernstein IL. 1996. Sodium appetite in the sham-drinking rat after chorda tympani nerve transection. Am $J$ Physiol 271:R339-345.

Fratucci De Gobbi JI, De Luca LA Jr, Johnson AK, Menani JV. 2001 Interaction of serotonin and cholecystokinin in the lateral parabrachial nucleus to control sodium intake. Am J Physiol Regul Integr Comp Physiol 280:R1301-1307.

Fulwiler CE, Saper CB. 1984. Subnuclear organization of the efferent connections of the parabrachial nucleus in the rat. Brain Res 319:229259

Gaykema RP, Chen CC, Goehler LE. 2007. Organization of immuneresponsive medullary projections to the bed nucleus of the stria termi- 
nalis, central amygdala, and paraventricular nucleus of the hypothalamus: evidence for parallel viscerosensory pathways in the rat brain. Brain Res 1130:130-145.

Geerling JC, Loewy AD. 2006a. Aldosterone-sensitive neurons in the nucleus of the solitary tract: bidirectional connections with the central nucleus of the amygdala. J Comp Neurol 497:646-657.

Geerling JC, Loewy AD. 2006b. Aldosterone-sensitive neurons in the nucleus of the solitary tract: efferent projections. J Comp Neurol 497:223250.

Geerling JC, Loewy AD. 2006c. Aldosterone-sensitive NTS neurons are inhibited by saline ingestion during chronic mineralocorticoid treatment. Brain Res 1115:54-64.

Geerling JC, Loewy AD. 2007. Sodium depletion activates the aldosteronesensitive neurons in the NTS independently of thirst. Am J Physiol Regul Integr Comp Physiol 292:R1338-1348.

Geerling JC, Engeland WC, Kawata M, Loewy AD. 2006a. Aldosterone target neurons in the nucleus tractus solitarius drive sodium appetite. J Neurosci 26:411-417.

Geerling JC, Kawata M, Loewy AD. 2006b. Aldosterone-sensitive neurons in the rat central nervous system. J Comp Neurol 494:515-527.

Gomez-Sanchez EP, Ganjam V, Chen YJ, Liu Y, Clark SA, Gomez-Sanchez CE. 2001. The 11beta hydroxysteroid dehydrogenase 2 exists as an inactive dimer. Steroids 66:845-848.

Hallbeck M, Hermanson O, Blomqvist A. 1999. Distribution of preprovasopressin mRNA in the rat central nervous system. J Comp Neurol 411:181-200

Hamilton RB, Norgren R. 1984. Central projections of gustatory nerves in the rat. J Comp Neurol 222:560-577.

Herbert H, Saper CB. 1990. Cholecystokinin-, galanin-, and corticotropinreleasing factor-like immunoreactive projections from the nucleus of the solitary tract to the parabrachial nucleus in the rat. J Comp Neurol 293:581-598.

Herbert H, Moga MM, Saper CB. 1990. Connections of the parabrachia nucleus with the nucleus of the solitary tract and the medullary reticular formation in the rat. J Comp Neurol 293:540-580.

Hermann GE, Rogers RC. 1985. Convergence of vagal and gustatory afferent input within the parabrachial nucleus of the rat. J Auton Nerv Syst 13:1-17.

Hermanson O, Blomqvist A. 1997. Preproenkephalin messenger RNAexpressing neurons in the rat parabrachial nucleus: subnuclear organization and projections to the intralaminar thalamus. Neuroscience 81:803-812.

Hochstenbach SL, Solano-Flores LP, Ciriello J. 1993. Fos induction in brainstem neurons by intravenous hypertonic saline in the conscious rat. Neurosci Lett 158:225-228.

Hoffman GE, Smith MS, Verbalis JG. 1993. c-Fos and related immediate early gene products as markers of activity in neuroendocrine systems. Front Neuroendocrinol 14:173-213.

Johnson AK, Thunhorst RL. 1997. The neuroendocrinology of thirst and salt appetite: visceral sensory signals and mechanisms of central integration. Front Neuroendocrinol 18:292-353.

Johnson AK, de Olmos J, Pastuskovas CV, Zardetto-Smith AM, Vivas L. 1999. The extended amygdala and salt appetite. Ann N Y Acad Sci 877:258-280.

Ju G, Swanson LW. 1989. Studies on the cellular architecture of the bed nuclei of the stria terminalis in the rat: I. Cytoarchitecture. J Comp Neurol 280:587-602.

Karimnamazi H, Travers SP, Travers JB. 2002. Oral and gastric input to the parabrachial nucleus of the rat. Brain Res 957:193-206.

Lane JM, Herbert J, Fitzsimons JT. 1997. Increased sodium appetite stimulates c-fos expression in the organum vasculosum of the lamina terminalis. Neuroscience 78:1167-1176.

Lu J, Sherman D, Devor M, Saper CB. 2006. A putative flip-flop switch for control of REM sleep. Nature 441:589-594.

Luppi PH, Aston-Jones G, Akaoka H, Chouvet G, Jouvet M. 1995. Afferent projections to the rat locus coeruleus demonstrated by retrograde and anterograde tracing with cholera-toxin B subunit and Phaseolus vulgaris leucoagglutinin. Neuroscience 65:119-160.

Menani JV, Johnson AK. 1998. Cholecystokinin actions in the parabrachial nucleus: effects on thirst and salt appetite. Am J Physiol 275:R14311437.

Menani JV, Thunhorst RL, Johnson AK. 1996. Lateral parabrachial nucleus and serotonergic mechanisms in the control of salt appetite in rats. Am J Physiol 270:R162-168.
Menani JV, De Luca LA Jr, Johnson AK. 1998. Lateral parabrachial nucleus serotonergic mechanisms and salt appetite induced by sodium depletion. Am J Physiol 274:R555-560.

Menani JV, Barbosa SP, De Luca LA Jr, De Gobbi JI, Johnson AK. 2002 Serotonergic mechanisms of the lateral parabrachial nucleus and cholinergic-induced sodium appetite. Am J Physiol Regul Integr Comp Physiol 282:R837-841.

Milner TA, Joh TH, Pickel VM. 1986. Tyrosine hydroxylase in the rat parabrachial region: ultrastructural localization and extrinsic sources of immunoreactivity. J Neurosci 6:2585-2603.

Morita H, Yamashita Y, Nishida Y, Tokuda M, Hatase O, Hosomi H. 1997. Fos induction in rat brain neurons after stimulation of the hepatoportal Na-sensitive mechanism. Am J Physiol 272:R913-923.

Norgren R. 1976. Taste pathways to hypothalamus and amygdala. J Comp Neurol 166:17-30.

Norgren R, Leonard CM. 1971. Taste pathways in rat brainstem. Science 173:1136-1139.

Norgren R, Pfaffmann C. 1975. The pontine taste area in the rat. Brain Res 91:99-117.

Norgren R, Smith GP. 1988. Central distribution of subdiaphragmatic vagal branches in the rat. J Comp Neurol 273:207-223.

Nose H, Mack GW, Shi XR, Nadel ER. 1988. Role of osmolality and plasma volume during rehydration in humans. J Appl Physiol 65:325-331.

Ohman LE, Johnson AK. 1986. Lesions in lateral parabrachial nucleus enhance drinking to angiotensin II and isoproterenol. Am J Physiol 251:R504-509.

Paues J, Mackerlova L, Blomqvist A. 2006. Expression of melanocortin-4 receptor by rat parabrachial neurons responsive to immune and aversive stimuli. Neuroscience 141:287-297.

Paxinos G, Watson C. 2005. The rat brain in stereotaxic coordinates. New York: Academic Press.

Prakash MR, Norgren R. 1991. Comparing salt appetites: induction with intracranial hormones or dietary sodium restriction. Brain Res Bull 27:397-401.

Reilly JJ, Maki R, Nardozzi J, Schulkin J. 1994. The effects of lesions of the bed nucleus of the stria terminalis on sodium appetite. Acta Neurobiol Exp (Wars) 54:253-257.

Ricardo JA, Koh ET. 1978. Anatomical evidence of direct projections from the nucleus of the solitary tract to the hypothalamus, amygdala, and other forebrain structures in the rat. Brain Res 153:1-26.

Rice KK, Richter CP. 1943. Increased sodium chloride and water intake of normal rats treated with desoxycorticosterone acetate. Endocrinology 33:106-115

Richter CP. 1936. Increased salt appetite in adrenalectomized rats. Am J Physiol 115:155-161.

Rinaman L, Verbalis JG, Stricker EM, Hoffman GE. 1993. Distribution and neurochemical phenotypes of caudal medullary neurons activated to express cFos following peripheral administration of cholecystokinin. J Comp Neurol 338:475-490.

Rinaman L, Baker EA, Hoffman GE, Stricker EM, Verbalis JG. 1998 Medullary c-Fos activation in rats after ingestion of a satiating meal. Am J Physiol 275:R262-268.

Roitman MF, Bernstein IL. 1999. Amiloride-sensitive sodium signals and salt appetite: multiple gustatory pathways. Am J Physiol 276:R17321738.

Roitman MF, Schafe GE, Thiele TE, Bernstein IL. 1997. Dopamine and sodium appetite: antagonists suppress sham drinking of $\mathrm{NaCl}$ solutions in the rat. Behav Neurosci 111:606-611.

Roland BL, Li KX, Funder JW. 1995. Hybridization histochemical localization of 11 beta-hydroxysteroid dehydrogenase type 2 in rat brain. Endocrinology 136:4697-4700.

Sagar SM, Sharp FR, Curran T. 1988. Expression of c-fos protein in brain: metabolic mapping at the cellular level. Science 240:1328-1331.

Sagar SM, Price KJ, Kasting NW, Sharp FR. 1995. Anatomic patterns of Fos immunostaining in rat brain following systemic endotoxin administration. Brain Res Bull 36:381-392.

Sakai RR, McEwen BS, Fluharty SJ, Ma LY. 2000. The amygdala: site of genomic and nongenomic arousal of aldosterone-induced sodium intake. Kidney Int 57:1337-1345.

Saper CB. 1996. Any way you cut it: a new journal policy for the use of unbiased counting methods. J Comp Neurol 364:5.

Saper CB, Loewy AD. 1980. Efferent connections of the parabrachial nucleus in the rat. Brain Res 197:291-317.

Scalera G, Spector AC, Norgren R. 1995. Excitotoxic lesions of the para- 
brachial nuclei prevent conditioned taste aversions and sodium appetite in rats. Behav Neurosci 109:997-1008.

Schreihofer AM, Anderson BK, Schiltz JC, Xu L, Sved AF, Stricker EM. 1999. Thirst and salt appetite elicited by hypovolemia in rats with chronic lesions of the nucleus of the solitary tract. Am J Physiol 276 : R251-258.

Sequeira SM, Geerling JC, Loewy AD. 2006. Local inputs to aldosteronesensitive neurons of the nucleus tractus solitarius. Neuroscience 141 : 1995-2005.

Shapiro RE, Miselis RR. 1985. The central neural connections of the area postrema of the rat. J Comp Neurol 234:344-364.

Stornetta RL, Norton FE, Guyenet PG. 1993. Autonomic areas of rat brain exhibit increased Fos-like immunoreactivity during opiate withdrawal in rats. Brain Res 624:19-28.

Stricker EM, Jalowiec JE. 1970. Restoration of intravascular fluid volume following acute hypovolemia in rats. Am J Physiol 218:191-196.

Stricker EM, Verbalis JG. 1996. Central inhibition of salt appetite by oxytocin in rats. Regul Pept 66:83-85.

Stricker EM, Thiels E, Verbalis JG. 1991. Sodium appetite in rats after prolonged dietary sodium deprivation: a sexually dimorphic phenomenon. Am J Physiol 260:R1082-1088.

Stricker EM, Bushey MA, Hoffmann ML, McGhee M, Cason AM, Smith JC. 2007. Inhibition of $\mathrm{NaCl}$ appetite when DOCA-treated rats drink saline. Am J Physiol Regul Integr Comp Physiol 292:R652-662.

Sugawara AM, Miguel TT, de Oliveira LB, Menani JV, De Luca LA Jr. 1999. Noradrenaline and mixed alpha 2-adrenoceptor/imidazolinereceptor ligands: effects on sodium intake. Brain Res 839:227-234.

Sunn N, McKinley MJ, Oldfield BJ. 2003. Circulating angiotensin II activates neurones in circumventricular organs of the lamina terminalis that project to the bed nucleus of the stria terminalis. J Neuroendocrinol 15:725-731.

Swank MW, Bernstein IL. 1994. c-Fos induction in response to a conditioned stimulus after single trial taste aversion learning. Brain Res 636:202-208

Thunhorst RL, Lewis SJ, Johnson AK. 1994. Effects of sinoaortic baroreceptor denervation on depletion-induced salt appetite. Am J Physiol 267:R1043-1049.

Travers SP. 2002. Quinine and citric acid elicit distinctive Fos-like immunoreactivity in the rat nucleus of the solitary tract. Am J Physiol Regul Integr Comp Physiol 282:R1798-1810.

Travers JB, Travers SP, Norgren R. 1987. Gustatory neural processing in the hindbrain. Annu Rev Neurosci 10:595-632.

van der Kooy D, Koda LY, McGinty JF, Gerfen CR, Bloom FE. 1984. The organization of projections from the cortex, amygdala, and hypothalamus to the nucleus of the solitary tract in rat. J Comp Neurol 224:1-24.
Wagman W. 1963. Sodium chloride deprivation: development of sodium chloride as a reinforcement. Science 140:1403-1404.

Wang T, Edwards GL. 1997. Differential effects of dorsomedial medulla lesion size on ingestive behavior in rats. Am J Physiol 273:R12991308.

Watson WE. 1985. The effect of removing area postrema on the sodium and potassium balances and consumptions in the rat. Brain Res 359:224232 .

Weisinger RS, Denton DA, McKinley MJ, Miselis RR, Park RG, Simpson JB. 1993. Forebrain lesions that disrupt water homeostasis do not eliminate the sodium appetite of sodium deficiency in sheep. Brain Res 628:166-178.

Willing AE, Berthoud HR. 1997. Gastric distension-induced c-fos expression in catecholaminergic neurons of rat dorsal vagal complex. Am J Physiol 272:R59-67.

Wolf G. 1964. Effect of dorsolateral hypothalamic lesions on sodium appetite elicited by desoxycorticosterone and by acute hyponatremia. J Comp Physiol Psychol 58:396-402.

Wolf G. 1965. Effect of deoxycorticosterone on sodium appetite of intact and adrenalectomized rats. Am J Physiol 208:1281-1285.

Wolf G. 1967. Hypothalamic regulation of sodium intake: relations to preoptic and tegmental function. Am J Physiol 213:1433-1438.

Wolf G, Handal PJ. 1966. Aldosterone-induced sodium appetite: doseresponse and specificity. Endocrinology 78:1120-1124.

Wotus C, Arnhold MM, Engeland WC. 2007. Dehydration-induced drinking results in a rapid decrease in Fos expression in hypothalamic paraventricular neurons expressing vasopressin, but not corticotropinreleasing hormone. Am J Physiol Regul Integr Comp Physiol 292: R1349-1358.

Xu YL, Reinscheid RK, Huitron-Resendiz S, Clark SD, Wang Z, Lin SH, Brucher FA, Zeng J, Ly NK, Henriksen SJ, de Lecea L, Civelli O. 2004. Neuropeptide S: a neuropeptide promoting arousal and anxiolytic-like effects. Neuron 43:487-497.

Yamamoto T, Shimura T, Sako N, Azuma S, Bai WZ, Wakisaka S. 1992. $\mathrm{C}$-fos expression in the rat brain after intraperitoneal injection of lithium chloride. Neuroreport 3:1049-1052.

Yamamoto T, Shimura T, Sako N, Sakai N, Tanimizu T, Wakisaka S. 1993 c-Fos expression in the parabrachial nucleus after ingestion of sodium chloride in the rat. Neuroreport 4:1223-1226.

Yoshida K, McCormack S, Espana RA, Crocker A, Scammell TE. 2005. Afferents to the orexin neurons of the rat brain. J Comp Neurol 494: $845-861$.

Zardetto-Smith AM, Beltz TG, Johnson AK. 1994. Role of the central nucleus of the amygdala and bed nucleus of the stria terminalis in experimentally-induced salt appetite. Brain Res 645:123-134. 